\title{
Duality and Modular Invariance in Rational Conformal Field Theories
}

\author{
Miao Li $i^{\star \star \star \star}$ \\ The Niels Bohr Institute, Blegdamsvej 17, DK-2100 Copenhagen Ø, Denmark
}

Received April 28, 1990; in revised form July 21, 1990

\begin{abstract}
We investigate the polynomial equations which should be satisfied by the duality data for a rational conformal field theory. We show that by these duality data we can construct some vector spaces which are isomorphic to the spaces of conformal blocks. One can construct explicitly the inner product for the former if one deals with a unitary theory. These vector spaces endowed with an inner product are the algebraic reminiscences of the Hilbert spaces in a Chern-Simons theory. As by-products, we show that the polynomial equations involving the modular transformations for the one-point blocks on the torus are not independent. We discuss the solution of structure constants for a physical theory. Making some assumption, we obtain a neat solution. And this solution in turn implies that the quantum groups of the left sector and of the right sector must be the same, although the chiral algebras need not be the same. Some examples are given. Finally, we discuss the reconstruction of the quantum group in a rational conformal theory.
\end{abstract}

\section{Introduction}

Despite the fact that much progress has been made in understanding the so-called rational conformal field theories (RCFT's) recently, there remain many open problems. Moore and Seiberg, in their work motivated by seeking a proof of the Verlinde conjecture [2], formulated an algebraic approach to RCFT's [3]. They refined the duality notations in the seminal work of BPZ [1] and obtained a whole set of polynomial equations for the duality data (see also [15] for a rigorous approach). Obviously, these data do not determine the conformal field theory completely: there are perhaps countably many models corresponding to a solution

* Address after October 1: Department of Physics, University of California, Santa Barbara, CA 91306, USA

$\star \star$ E-mail address: LI@ voodow. bidnet 
to these polynomial equations ${ }^{1}$. Thus, classifying RCFT's must consist in two steps, the first step is to classify all solutions to the polynomial equations, the second one is to reconstruct from a solution the whole conformal field theory.

Let us concentrate our attention on the first step. The basic duality data introduced by Moore and Seiberg and others [4] are the fusing matrices, the monodromy phases of three-point conformal blocks and the modular transformations $S(n)$ for the one-point blocks on the torus. It was possible to eliminate the latter as the basic data [5] and further in [6] it was shown that the polynomial equations involving these data are not independent. However, the modular property of the characters has not been eliminated from the elementary equations. On the other hand, if one believes that to every solution of the duality data one can associate a quantum group, this property is automatically satisfied for all known quantum groups. Therefore, we believe that the elementary equations are those involving only the fusing matrices and the braiding matrices. [6] is based on the technique that various vector spaces can be represented by graphs. These vector spaces are available in the Chern-Simons theory (CSGT) [7]. It is still a conjecture that a rational conformal field theory corresponds to a Chern-Simons theory. So to base [6] on a solid foundation we need to construct these vector spaces for any rational conformal field theory. This is one of the purposes of the present work. Our second purpose is to solve equations concerning structure constants, which combine the left sector and the right sector. These equations are overdetermined. We show that there are simple solutions and conjecture that these are only solutions. Finally, we generalize Witten's construction of quantum group in $S U(2)$ WZW model to more general cases, using the so-called lassoing procedure.

To start with, we give a brief summary of the present status of the algebraic approach to RCFT's in Sect. 2. Duality properties are described. We also present a revised algebraic axiom system for duality data. We eliminate identities for $S(n)$, but supplement with the definition of $S(n)$. For the original algebraic axiom system, please see [3].

In studying the polynomial identities, perhaps the most basic problem is to choose an appropriate "gauge" of chiral vertices in which some duality data take simple form. We shall do gauge fixing in Sect. 3. There are at least two advantages with this gauge. First, elements of fusing matrices, when represented by closed graphs, take simpler form. This enables us to prove the tetrahedron symmetry, which in turn proves very useful in proving independence of identities involving $S(n)$ and in solving identities involving structure constants, namely the coefficients used to combine the left sector to the right sector. Second, such a normalization of elements of fusing matrices allows us to compare directly to the normalization introduced in the framework of the Chern-Simons gauge theory [7] and proves useful in study of the quantum group. Some facts concerning the normalization of fusing matrices chosen in our construction are undoubtedly known to some experts, but have not been exposed systematically.

Construction of various types of graphs from duality data was introduced in [15] and [14]. Reidemeister moves (taking braiding phases into account) were verified mainly in [14]. In Sect. 4, we shall construct tetrahedra representing elements of fusing matrices based on these works. We also prove tetrahedron symmetries there. Although it is widely believed that there should be such symmetries, this is perhaps the first time to prove them. Construction of open

${ }^{1}$ I would like to thank V. Fateev for a discussion on this point 
graphs then inspires us to construct various vector spaces in Sect. 5, these vector spaces in some sense are isomorphic to various Hilbert spaces in the CSGT, therefore isomorphic to spaces of conformal blocks in the RCFT. We show that for a unitary RCFT, we can define an inner product for the vector space. The inner products are represented by closed graphs. These graphs are needed in proving independence of identities involving $S(n)$ in [6]. For an illustration, we present the proof for one of them in Sect. 6. We supplement Proposition 5 to show that $S(n)$ (together with $T$ ) actually generates a projective representation of the modular group of genus one.

In Sect. 7, using the gauge fixed in Sect. 3 and tetrahedron symmetries, we give a solution for structure constants. However, at present we are unable to eliminate an assumption for couplings of chiral vertices, which is not necessary when all fusion rules $N_{j k}^{i} \leqq 1$. The main result is that there should be an automorphism among fusing matrices in two sectors. In our previous work [5], we obtained a less strong similar result. This result suggests that our assumption is reasonable. We give several examples at the end of Sect. 7.

Our second purpose of this paper is to reconstruct the quantum group [8]. It is generally believed that for each solution to the polynomial equations, there is a hidden quantum group. By investigating the monodromy property of solutions to the Knizhnik-Zamolodchikov equations, one finds that the universal $\mathscr{R}$-matrix naturally appears as the monodromy matrix [9]. Alvarez-Gaumé et al. argued that the quantum group as an algebra is the centralizer algebra of the braid groups acting on the spaces of conformal blocks [10]. Moore and Reshetikhin proposed some new chiral vertex operators and the quantum group acts on these operators naturally [11]. In their construction, one has to invoke some so-called phenomenological observations. Still, a conceptual explanation is lacking. Starting from the Chern-Simons theory, Witten has been able to define the relations among generators of the quantum group by the so-called lassoing procedure [12]. The drawback is that one must work with a Chern-Simons theory.

In [13], Alvarez-Gaumé et al. defined another kind of quantum group, which we shall call the IRF quantum group. It has a close relationship with the quantum group in the original sense (which sometimes we call the vertex quantum group). We show in Sect. 8 that the algebra defined in [13] is actually a quasitriangular Hopf algebra, the alternative name of the quantum group (a short introduction to quasitriangular Hopf algebras is given in Appendix A). We also show that candidates for the irreducible representation spaces of this algebra, by definition, also obey restricted tensor product property. This indicates its relation to the vertex quantum group, because in the representation theory of the vertex quantum group, usual notation of tensor products must be replaced by the one of restricted tensor product when the deformation parameter is a root of unity. Based on this observation, we generalize Witten's lassoing procedure for an arbitrary WZW model, we give a systematic approach which applies to any WZW models. The quantum groups obtained in this way are the ones one expects. For example, for the $S U(2)$ WZW model, the quantum group is $U_{q}(S U(2))$. Note that Witten worked out the quantum group which is dual to $U_{q}(S U(2))$ by a special prescription. We just give some evidence for the existence of some limits of the duality data, instead of proving it. However, we give two examples in which the lassoing procedure works. In Appendix B, we show that if the existence of these limits is granted, what we have constructed is actually the quantum group. Perhaps the most important thing which needs to be done is to construct the quantum group without using the Witten's lassoing. In performing Witten's lassoing, one has to analytically extend 
some parameters to the complex domain and take an infinite limit of certain indices for some duality data. However, our work on relating the IRF and vertex quantum groups strongly suggests that it is possible to construct the vertex quantum group from the duality data without invoking the limiting procedure. This remains a problem in our future work [21].

\section{Some Preliminaries for RCFT's}

Let $\mathscr{H}$ be the Hilbert space of a rational conformal field theory in which the chiral algebra $\mathscr{A} \otimes \overline{\mathscr{A}}$ is assumed. Here we use $\mathscr{A}$ to denote the holomorphic part and $\overline{\mathscr{A}}$ the anti-holomorphic part. The term rational is due to the finiteness of the decomposition of $\mathscr{H}$ into irreducible representations of $\mathscr{A} \otimes \overline{\mathscr{A}}$ :

$$
\mathscr{H}=\oplus_{(i, i)} \mathscr{H}_{i} \otimes \overline{\mathscr{H}}_{i},
$$

where $\mathscr{H}_{i}$ and $\overline{\mathscr{H}}_{i}$ are irreducible representations of algebras $\mathscr{A}$ and $\overline{\mathscr{A}}$ respectively. In particular, we identify $\mathscr{H}_{0}$ with $\mathscr{A}$ and $\overline{\mathscr{H}}_{0}$ with $\overline{\mathscr{A}}$.

We concentrate our attention on the holomorphic part, but the results are also valid for the anti-holomorphic part. The correlation functions on the sphere decompose into terms factorizing into holomorphic and anti-holomorphic parts. The independent holomorphic factors are named conformal blocks and can be built from the chiral vertices. The chiral vertex is a certain coupling among three representation spaces of $\mathscr{A}$. Here we use the notation of Fröhlich et al. We will not repeat the axioms $[3,15]$ in defining the chiral vertices which are linear maps

$$
\mathscr{H}_{i} \otimes_{z} \mathscr{H}_{j} \rightarrow \mathscr{H}_{k} .
$$

We denote the space of all such maps by $V_{j i}^{k}$ with the finite dimension $N_{j i}^{k}$, this is the fusion rule. A chiral vertex is specified by linear maps

$$
V_{\alpha}^{k i}\left(\left|\xi_{j}\right\rangle, z\right): \mathscr{H}_{i} \rightarrow \mathscr{H}_{k},
$$

where $\left|\xi_{j}\right\rangle \in \mathscr{H}_{j}$.

Suppose that we have a one to one map from the set (i) to itself, $i \rightarrow i^{\vee}, i^{\vee}$ is the charge conjugate of $i$. Let $|0\rangle$ be the vacuum. Then we have the following properties of the chiral vertices:

1. There is only one coupling

$$
V_{0}^{0 i}: \mathscr{H}_{i} \bigotimes_{z} \mathscr{H}_{i^{\vee}} \rightarrow \mathscr{H}_{0} .
$$

Here we use 0 to denote the unique vertex.

2. We have

$$
\mathscr{H}_{i} \bigotimes_{z}|0\rangle \cong|0\rangle \otimes_{z} \mathscr{H}_{i} \cong \mathscr{H}_{i}
$$

such that

$$
V_{0}^{i i}(|0\rangle, z)\left|\xi_{i}\right\rangle=V_{0}^{i 0}\left(\left|\xi_{i}\right\rangle, 0\right)|0\rangle=\left|\xi_{i}\right\rangle .
$$

The last two equations give a normalization of corresponding vertices involved.

Let $\left|\xi_{i}^{N}\right\rangle$ be a basis for $\mathscr{H}_{i}$. By property 1 , we can choose a basis $\left|\xi_{i^{\vee}}^{N}\right\rangle$ for $\mathscr{H}_{i^{\vee}}$ such that

$$
\left\langle\xi_{i}^{N}\right|=\lim _{z \rightarrow \infty}\langle 0| V_{0}^{0 i}\left(\left|\xi_{i}^{N}\right\rangle, z\right) z^{2 h\left(\xi_{i}^{N}\right)} .
$$


Here $\left(\left\langle\xi_{i}^{N}\right|\right)$ is dual to $\left(\left|\xi_{i}^{N}\right\rangle\right)$. This also fixes the normalization of the vertex $V_{0}^{0 i}$ once the bases for $\mathscr{H}_{i}$ and $\mathscr{H}_{i}$ v are chosen. as

We come to the duality properties. Define the braiding matrices $B\left[\begin{array}{ll}j & l \\ i & k\end{array}\right]( \pm)$

$$
\left[V_{\alpha}^{i m}\left(\left|\xi_{j}\right\rangle, z_{1}\right) V_{\beta}^{m k}\left(\left|\xi_{l}\right\rangle, z_{2}\right)\right]_{ \pm}=\sum_{n, \gamma, \delta} B_{m n}\left[\begin{array}{ll}
j & l \\
i & k
\end{array}\right]_{\alpha \beta}^{\gamma \delta}( \pm) V_{\gamma}^{i n}\left(\left|\xi_{l}\right\rangle, z_{2}\right) V_{\delta}^{n k}\left(\left|\xi_{j}\right\rangle, z_{1}\right),
$$

where signs \pm are used to denote two ways of analytic continuation of operator $V_{\alpha}^{i m}\left(\left|\xi_{j}\right\rangle, z_{2}\right) V_{\beta}^{m k}\left(\left|\xi_{l}\right\rangle, z_{1}\right)$ to $V_{\alpha}^{i m}\left(\left|\xi_{j}\right\rangle, z_{1}\right) V_{\beta}^{m k}\left(\left|\xi_{l}\right\rangle, z_{2}\right)$. Equation (2.4) states that the dimensions of spaces $\oplus_{m} V_{j m}^{i} \otimes V_{l k}^{m}$ and $\oplus_{n} V_{l n}^{i} \otimes V_{j k}^{n}$ are the same, namely $\sum_{m} N_{j m}^{i} N_{l k}^{m}$ $=\sum_{n} N_{l n}^{i} N_{j k}^{n}$. Pictorially, we can rewrite (2.4) as

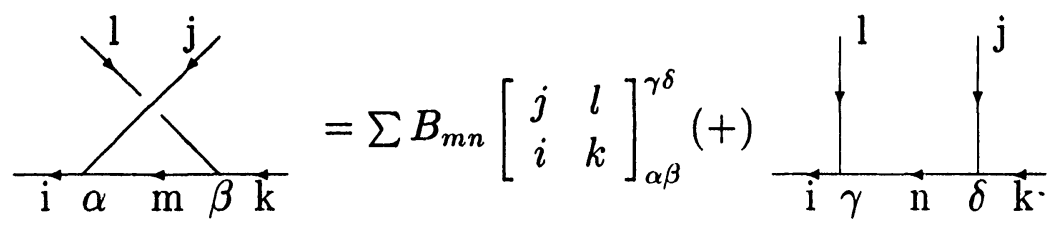

The fusing matrices can be defined as the structure constants of products of chiral vertices:

$$
\begin{aligned}
& V_{\alpha}^{i m}\left(\left|\xi_{j}\right\rangle, z_{1}\right) V_{\beta}^{m k}\left(\left|\xi_{l}\right\rangle, z_{2}\right) \\
& \quad=\sum_{n, \gamma, \delta} F_{m n}\left[\begin{array}{ll}
j & l \\
i & k
\end{array}\right]_{\alpha \beta}^{\gamma \delta} \sum_{N}\left\langle\xi_{n}^{N}\right| V_{\gamma}^{n l}\left(\left|\xi_{j}\right\rangle, z_{1}-z_{2}\right)\left|\xi_{l}\right\rangle V_{\delta}^{i k}\left(\left|\xi_{n}^{N}\right\rangle, z_{2}\right) .
\end{aligned}
$$

This again means that the dimensions of two spaces $\oplus_{m} V_{j m}^{i} \otimes V_{l k}^{m}$ and $\oplus_{n} V_{j l}^{n} \otimes V_{n k}^{i}$ are same. The graphic representation of (2.6) is
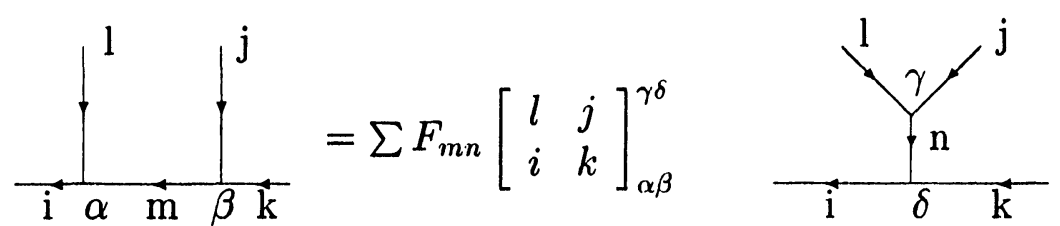

Note that objects represented by graphs in (2.5) and (2.7) are linear independent, so both $B( \pm)$ and $F$ as matrices are invertible. So far we have used open graphs with vertices of $\phi^{3}$ type to represent the conformal blocks. We will not do so further, however, we shall use these graphs to represent quantities constructed from $B$ 's and $F$ 's, following Fröhlich and King.

Finally, notice that $F_{i k^{\vee}}\left[\begin{array}{cc}i^{\vee} & j \\ 0 & k\end{array}\right]_{0 \alpha}^{\beta 0}$, as matrix with indices $\alpha$ and $\beta$, is invertible. For the spaces $V_{j k}^{i}$ and $V_{i}^{k} v_{j}$, we can choose bases such that

$$
F_{i k^{\vee}}\left[\begin{array}{cc}
i^{\vee} & j \\
0 & k
\end{array}\right]_{0 \alpha}^{\beta 0}=\delta_{\alpha}^{\beta}
$$

This equation fixes the relative normalization of bases for $V_{j k}^{i}$ and $V_{i}^{k_{j}}$. 
Finally, we give the algebraic axiom system for the duality data of a RCFT [3]. The duality data are

D1. A set (i) with a one-to-one map: $i \rightarrow i^{\vee}$, the charge conjugation. We therefore define the charge conjugation matrix by $C_{i j}=\delta_{i j}$. The fusion rules $N_{j k}^{i}$ form a commutative and associative fusion algebra [2]. In addition, $N_{i j}^{0}=C_{i j}$.

D2. Fusing matrices:

$$
\oplus_{m} V_{j m}^{i} \otimes V_{l k}^{m} \stackrel{F}{\longrightarrow} \oplus_{n} V_{j l}^{n} \otimes V_{n k}^{i} .
$$

D3. The maps $\Omega( \pm): V_{i j}^{k} \rightarrow V_{j i}^{k}$, which are given by phases

$$
\varepsilon_{i j}^{k}(\alpha) e^{ \pm i \pi\left(h_{k}-h_{i}-h_{j}\right)} \text {. }
$$

$\varepsilon_{i j}^{k}(\alpha)$ are signs. Hence the conformal weights are determined up to integers.

The braiding matrices are not independent data [3]. Similarly, the modular transformations of the characters and the one-point blocks on the torus are not independent either. They are defined by M1.

where

$$
\begin{aligned}
\frac{S_{i, j}}{S_{0,0}} & =\frac{1}{F_{i} F_{j}}\left(B\left[\begin{array}{cc}
j & i \\
j & i^{\vee}
\end{array}\right](-) B\left[\begin{array}{cc}
i & j \\
j & i^{\vee}
\end{array}\right](-)\right)_{00}^{00} \\
& =\sum_{q} e^{i 2 \pi\left(h_{q}-h_{i}-h_{j}\right)}\left(F_{q}\right)^{-1} N_{i j}^{q}
\end{aligned}
$$

$$
F_{q}=F_{00}\left[\begin{array}{cc}
q & q^{\vee} \\
q & q
\end{array}\right]_{00}^{00}
$$

M2. Similarly

$$
S(j)^{i \alpha, l \beta}=\sum_{q, \gamma} e^{i 2 \pi\left(h_{q}-h_{i}-h_{l}\right)} S_{0, q} F_{l i}\left[\begin{array}{ll}
j & i \\
l & q
\end{array}\right]_{\beta \gamma}^{\alpha \gamma},
$$

where we have used the famous relation [3]

$$
F_{q}=\frac{S_{0,0}}{S_{0, q}} .
$$

The formula for $S_{i, j}$ was obtained in $[3,17]$ and formula for $S(j)$ was first obtained in [5].

Now the constraint equations are

C1. Pentagon identity

$$
\tau_{23} F_{13} F_{12}=F_{23} F_{12} F_{23},
$$

the detailed form is written in (3.1).

C2. Two hexagon identities

$$
F(\Omega( \pm) \otimes 1) F=(1 \otimes \Omega( \pm)) F(1 \otimes \Omega( \pm)) .
$$

C3. $\left(S T S^{-1}\right)_{0, j}=\left(T^{-1} S^{-1} T^{-1}\right)_{0, j}$.

Remark. In [6], using M2 and C3, we proved the independence of identities involving $S(j)$ introduced by Moore and Seiberg in [3]. Since the proof is based on graph manipulations, we shall present a proof for one of those identities in Sect. 6. 
For notations in $\mathrm{C1}-3$, see [3]. Note that combining the pentagon identity one can replace two hexagon identities by two Yang-Baxter equations. All these equations together with definitions $\mathrm{M} 1$ and $\mathrm{M} 2$ guarantee that we have a consistent algebraic system with properties of duality and modular covariance for any genus. By D1 and C3, it is possible to prove the Verlinde conjecture $[2,3,17$, 10] and the contraints $(S T)^{3}=S^{2}=C$, where $C$ is the charge conjugation matrix. We will show this in Sect. 6. In the framework of quantum groups, Alvarez-Gaumé et al. claimed that one can lift $\mathrm{C} 3$ for all known quantum group [10]. It has a natural explanation in terms of the quantum group. So we suspect that whether C3 is really independent of the rest of the constraints or not. It is interesting to see that only in C3 the central charge appears via $T=\operatorname{diag}\left(\exp i 2 \pi\left(h_{i}-\frac{c}{24}\right)\right)$.

\section{Normalization of Fusing Matrices}

In this section we shall discuss the "gauge fixing" of the fusing matrices. There are two purposes for doing this. First, to construct the closed graphs which represent certain duality data in a neat way, which must be similar to the way in which Witten defined normalization of graphs in a Chern-Simons theory. We will see that for a general rational conformal field theory, one can obtain a normalization similar to the one given by Witten. Second, to have a convenient normalization will prove helpful in solving equations involving structure constants (in the sense of Moore and Seiberg). Note that several authors have used the normalization we will discuss for models with $N_{j k}^{i} \leqq 1$. But a full discussion for the general cases has not been given.

\subsection{Some Useful Formulae}

To begin with, we write down the general form of the pentagon identities

$$
\begin{aligned}
\sum_{\beta} F_{p_{3} r_{2}} & {\left[\begin{array}{cc}
r_{1} & k \\
p_{1} & p_{4}
\end{array}\right]_{\beta \alpha_{3}}^{\gamma_{2} \gamma_{3}} F_{p_{2} r_{1}}\left[\begin{array}{cc}
i & j \\
p_{1} & p_{3}
\end{array}\right]_{\alpha_{1} \alpha_{2}}^{\gamma_{1} \beta} } \\
& =\sum_{s, \beta_{i}} F_{p_{3} s}\left[\begin{array}{cc}
j & k \\
p_{2} & p_{4}
\end{array}\right]_{\alpha_{2} \alpha_{3}}^{\beta_{1} \beta_{2}} F_{s r_{1}}\left[\begin{array}{cc}
i & j \\
r_{2} & k
\end{array}\right]_{\beta_{3} \beta_{1}}^{\gamma_{1} \gamma_{2}} F_{p_{2} r_{2}}\left[\begin{array}{cc}
i & s \\
p_{1} & p_{4}
\end{array}\right]_{\alpha_{1} \beta_{2}}^{\beta_{3} \gamma_{3}} .
\end{aligned}
$$

We give the following two special cases which will prove useful later. First, let $p_{2}=r_{2}=0$, we find only when $p_{3}=j^{\vee}, p_{1}=p_{4}=i, r_{1}=k^{\vee}$ the corresponding $F$ 's in the left-hand side of the above formula are not zero. In addition, the couplings $\alpha_{1}$, $\alpha_{2}, \gamma_{2}$, and $\gamma_{3}$ are unique, which we can denote by 0 . Similarly, in the right-hand side of the formula, $s=i^{\vee}$ and $\beta_{2}=0, \beta_{1}=\alpha_{3}$. Finally, we use the formulae

$$
F_{j^{\vee} i^{\vee}}\left[\begin{array}{cc}
j & k \\
0 & i
\end{array}\right]_{0 \alpha_{3}}^{\alpha_{3} 0}=F_{i^{\vee} k^{\vee}}\left[\begin{array}{ll}
i & j \\
0 & k
\end{array}\right]_{0 \alpha_{3}}^{\alpha_{3} 0}=1
$$

which come from (2.8) to obtain the special pentagon identities

$$
\sum_{\beta} F_{0 k^{\vee}}\left[\begin{array}{cc}
i & j \\
i & j^{\vee}
\end{array}\right]_{00}^{\gamma_{1} \beta} F_{j^{\vee} 0}\left[\begin{array}{cc}
k^{\vee} & k \\
i & i
\end{array}\right]_{\beta \alpha_{3}}^{00}=\delta_{\gamma_{1} \alpha_{3}} F_{00}\left[\begin{array}{cc}
i & i^{\vee} \\
i & i
\end{array}\right]_{00}^{00} .
$$


For fixed indices $i, j, k$, above equation states that as matrices

$$
F_{0 k}\left[\begin{array}{cc}
i & j^{\vee} \\
i & j
\end{array}\right] \text { and } F_{j 0}\left[\begin{array}{cc}
k & k^{\vee} \\
i & i
\end{array}\right]
$$

are nonsingular provided $F_{00}\left[\begin{array}{cc}i & i^{\vee} \\ i & i\end{array}\right]=F_{i}$ is not zero. Here we have replaced $j^{\vee}$ and $k^{\vee}$ by $j$ and $k$ respectively. The fact that $F_{i}$ cannot be zero and $F_{i^{\vee}}=F_{i}$ is proved in $[2,3]$. For a unitary theory $F_{i}>0$.

In (3.1), let $p_{3}=r_{2}=0$, in this case the left-hand side gives $F_{i}$, and we find another useful formula

$$
\sum_{\beta} F_{0 k^{\vee}}\left[\begin{array}{cc}
j & i^{\vee} \\
j & i
\end{array}\right]_{00}^{\alpha \beta} F_{j 0}\left[\begin{array}{cc}
k & k^{\vee} \\
i & i
\end{array}\right]_{\gamma \beta}^{00}=\delta_{\alpha \gamma} F_{i}
$$

Comparing (3.3) with (3.4), we deduce

$$
F_{0 k^{\vee}}\left[\begin{array}{cc}
j & i^{\vee} \\
j & i
\end{array}\right]_{00}^{\beta \alpha}=F_{0 k}\left[\begin{array}{cc}
i & j^{\vee} \\
i & j
\end{array}\right]_{00}^{\alpha \beta}
$$

Again in (3.1), let $p_{2}=r_{1}=0$, we find

$$
\sum_{\beta} F_{0 i}\left[\begin{array}{cc}
k & j \\
k & j^{\vee}
\end{array}\right]_{00}^{\beta \alpha} F_{j 0}\left[\begin{array}{cc}
k & k^{\vee} \\
i & i
\end{array}\right]_{\beta \gamma}^{00}=\delta_{\alpha \gamma} F_{k} .
$$

Combining this with (3.4) and (3.5), we obtain an important formula,

$$
F_{0 k}\left[\begin{array}{cc}
i & j^{\vee} \\
i & j
\end{array}\right]^{T} / F_{i}=F_{0 i}\left[\begin{array}{cc}
k & j \\
k & j^{\vee}
\end{array}\right] / F_{k},
$$

where by the superscript $T$ we mean matrix transposition. This formula will be relevant in our gauge fixing of the $F$ 's, which in turn implies tetrahedron symmetry of the $F$ 's.

\subsection{Gauge Fixing}

We first prove the following theorem.

Theorem 1. For the unitary theory, the matrix $F_{0 i}\left[\begin{array}{cc}j & k^{\vee} \\ j & k\end{array}\right]$ is a non-singular $N_{i k}^{j} \times N_{i k}^{j}$ (complex) matrix, and its entry is nonvanishing only when indices $\alpha$ and $\beta$ are such that signs $\varepsilon_{j k^{v}}^{i}(\alpha)$ and $\varepsilon_{i k}^{j}(\beta)$ are same.

Proof. The first fact can be easily learned from (3.4) or (3.5), since for a unitary model, $F_{i}$ is not zero. To prove the second fact, consider the following relation:

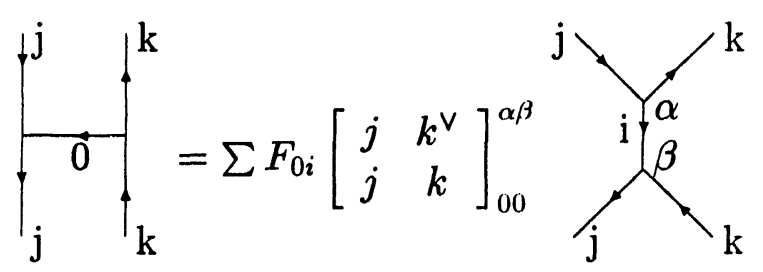


This relation can be viewed as the fusing relation for conformal blocks, or the relation among quantities constructed from duality data. For the latter explanation, see [14] and Sect. 5 in this paper. It is convenient to work with the latter explanation. Doing braiding twice on both sides of the above equation (amounts to multiplying braiding matrix twice in the second explanation), we have

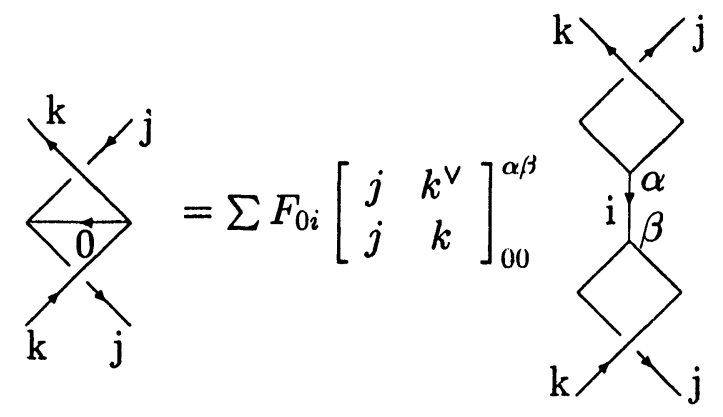

It has been proved in [14] that the quantity in the left-hand side of the above equation is just<smiles>[Y]C([Y])C(=O)C(C)C</smiles>

while the quantity in the right-hand side of the above equation represented by that graph is given by the similar graph on the right-hand side of (3.8) with two extra phases, finally we find

$$
\left\{_{\mathrm{k}}^{\mathrm{k}}\right\}_{\mathrm{j}}^{\mathrm{j}}=\sum F_{0 i}\left[\begin{array}{cc}
j & k^{\vee} \\
j & k
\end{array}\right]_{00}^{\alpha \beta} \epsilon_{j k^{\vee}}^{i}(\alpha) \epsilon_{i k}^{j}(\beta)
$$

Recall that relation (3.8) and (3.9) must be the same, this is due to the fact that the order of indices $j$ and $k$ is irrelevant in our case, since there exists an involution $z \rightarrow 1 / \bar{z}$ (roughly speaking, this involution exchanges points 0 and $\infty$ on the Riemann sphere, thus (3.8) and (3.9) is the same as explained as relations among conformal blocks. This result can be readily generalized to the other explanation). Equating (3.8) and (3.9), we find $\varepsilon_{j k^{k}}^{i}(\alpha) \varepsilon_{i k}^{j}(\beta)=1$ for a nonvanishing entry of the fusing matrix.

Remark. Similar to (3.5), we can prove

$$
F_{0 i}\left[\begin{array}{cc}
k^{\vee} & j \\
k^{\vee} & j^{\vee}
\end{array}\right]_{00}^{\alpha \beta}=F_{0 i}\left[\begin{array}{cc}
j & k^{\vee} \\
j & k
\end{array}\right]_{00}^{\alpha \beta}
$$

by using the pentagon identity. The quantity on the left-hand side in the above equation is just the coefficient on the right-hand side of (3.9). This can replace the argument in the last paragraph. 
Now we shall show that by choosing a certain "gauge" for the chiral vertices, these matrices can be diagonalized into the form

$$
F_{0 i}\left[\begin{array}{cc}
j & k^{\vee} \\
j & k
\end{array}\right]=F_{j k}^{i} e
$$

with appropriate constants $F_{j k}^{i}$, where $e$ is the unit matrix. Surprisingly, in the generic gauge, $F_{j k}^{i}=\left(F_{j} F_{k} / F_{i}\right)^{1 / 2}$, the same form given by Witten in the ChernSimons gauge theory!

For the space $V_{i k}^{j}$, we do a "gauge transformation" given by a $N_{i k}^{j} \times N_{i k}^{j}$ complex non-singular matrix $A_{i k}^{j}$, namely

$$
\tilde{V}_{\alpha}^{j k}\left(\left|\xi_{i}\right\rangle, z\right)=\sum_{\beta}\left(A_{i k}^{j}\right)^{\alpha \beta} V_{\beta}^{j k}\left(\left|\xi_{i}\right\rangle, z\right)
$$

We shall use $A_{i k}^{j}$ to diagonalize $F_{0 i}\left[\begin{array}{cc}j & k^{\vee} \\ j & k\end{array}\right]$ which is block diagonalized, according to Theorem 1. Therefore, we also require that $A_{i k}^{j}$ is block diagonalized, namely, under $A_{i k}^{j}$, chiral vertices $V_{\alpha}^{j k}$ with positive $\varepsilon_{i k}^{j}(\alpha)$ do not mix with ones with negative $\varepsilon_{i k}^{j}(\alpha)$. This is also quite physical, otherwise after gauge transformation, braiding matrix may have no definite eigenvalues with new chiral vertices.

Since we have already fixed the normalization of some chiral vertices, the $A_{i k}^{j}$,s cannot be arbitrary, they are subject to

$$
A_{i 0}^{i}=A_{i{ }^{v}}^{0}=1,
$$

coming from property 2 in the last section, and

$$
A_{i k}^{j}=A_{j^{\vee} i}^{k^{\vee}}
$$

from Eq. (2.8).

Under the "gauge transformation," we have

$$
F_{0 i}\left[\begin{array}{cc}
j & k^{\vee} \\
j & k
\end{array}\right]=\left(A_{j k^{\vee}}^{i}\right)^{T} \tilde{F}_{0 i}\left[\begin{array}{cc}
j & k \\
j & k
\end{array}\right] A_{i k}^{j}
$$

where we used matrix notation. By Theorem 1, all matrices in (3.14) are block diagonalized in the same fashion. We see that under these transformations, $F_{i}$ is gauge invariant. From (3.14) one may think that $F_{0 i}\left[\begin{array}{cc}j & k^{\vee} \\ j & k\end{array}\right]$ can be diagonalized into an arbitrary form, this is not the case as we shall see. First suppose all these matrices are diagonalized, Eq. (3.13) then implies that we shall have

$$
F_{0 i}\left[\begin{array}{cc}
j & k^{\vee} \\
j & k
\end{array}\right] \propto F_{0 j^{\vee}}\left[\begin{array}{cc}
k^{\vee} & i^{\vee} \\
k^{\vee} & i
\end{array}\right]
$$

One easily sees that this is a consequence of Eqs. (3.5) and (3.7). Therefore there is no obstruction coming from (3.15) [or (3.13)] to the simultaneous diagonalization. Next we shall note that another matrix under the transformation involving $A_{i k}^{j}$ is $F_{0 j}\left[\begin{array}{cc}i & k \\ i & k^{\vee}\end{array}\right]$, we have

$$
F_{0 j}\left[\begin{array}{cc}
i & k \\
i & k^{\vee}
\end{array}\right]=\left(A_{i k}^{j}\right)^{T} \tilde{F}_{0 j}\left[\begin{array}{cc}
i & k \\
i & k^{\vee}
\end{array}\right] A_{j k^{\vee}}^{i}
$$

which also contains $A_{j k}^{i}$. 
Now suppose a certain $F$-matrix is diagonalized as in (3.10). We find from (3.16) and (3.7) that $F_{0 j}\left[\begin{array}{cc}i & k \\ i & k^{\vee}\end{array}\right]$ is also diagonalized, and

$$
F_{i k^{v}}^{j} / F_{j k}^{i}=F_{i} / F_{j} \text {. }
$$

This is only a restriction on possible $F_{j k}^{i}$. It means that $F_{j k}^{i}$ cannot be arbitrary. A generic solution of (3.17) is $F_{i k^{\vee}}^{j}=f_{j}^{i} f_{k^{\vee}}$ and $f_{k^{\vee}}=f_{k^{1}}$. From (3.17) we find $f_{j}^{i} / f_{i}^{j}$ $=F_{i} / F_{j}$, and a typical choice of $f_{j}^{i}$ is $f_{j}^{i}=\left(F_{i} / F_{j}\right)^{1 / 2}$. Finally note that $F_{0 i}^{i}=F_{i}$ by definition, so $f_{k}=\sqrt{F_{k}}$. To summarize, we have proved the following theorem:

Theorem 2. It is always possible to choose a gauge for chiral vertices such that certain fusing matrices can be diagonalized as in (3.10) with

$$
F_{i k}^{j}=\left(F_{i} F_{k} / F_{j}\right)^{1 / 2} \text {, }
$$

and in the new gauge, braiding matrices have definite eigenvalues with new chiral vertices.

Notice that (3.18) is the same as suggested by Witten in the framework of Chern-Simons theory. We have not assumed $F_{i}>0$. Equation (3.18) must be understood as a product of various factors $\sqrt{F_{i}}$.

Remark. The gauge (3.18) is almost implied by our natural normalization for some special chiral vertices set in Sect. 2. If we do not assume this normalization, then $A_{i 0}^{i}$ and $A_{i}^{0{ }^{v_{i}}}$ can be arbitrary, now any solution must obey the following restriction:

$$
F_{i k^{\vee}}^{j} / F_{j k}^{i}=F_{j} A_{j 0}^{j} / F_{i} A_{i 0}^{i} .
$$

especially we can choose $A_{i 0}^{i}$ such that in (3.18) every $F_{i}$ is replaced by its absolute value.

Finally, we note that (3.18) does not fix the gauge completely. We have residual gauge transformations which are subject to

$$
\left(A_{i k}^{j}\right)^{T} A_{j k}^{i}=e, \quad A_{i k}^{j}=A_{j^{\vee} v_{i}}^{k^{\vee}} .
$$

These will prove useful in Sect. 7.

\section{Construction of Tetrahedra}

The tetrahedron graphs can easily be constructed both within frameworks of quantum group theory and Chern-Simons theory. The construction for a general rational conformal field theory is believed to exist, but nobody has given such a construction. What we will give here is a straightforward consequence of the work of Fröhlich and King [14]. By the duality property, one can show that there are tetrahedron symmetries among entries of the fusing matrices. Both the tetrahedra and the tetrahedron symmetries are used in the proof of the independence of the polynomial equations involving $S(n)$.

\subsection{Definition of Graphs}

We shall make use of the elegant pictorial representations of the basic data $B$ 's and $F$ 's given in $[14,15]$. For simplicity, we assume $N_{j k}^{i} \leqq 1$ in this section. The results 
obtained can be generalized straightforwardly to the other cases. We will first review the definitions of fundamental graphs, following $[15,14]$. All topological moves have been proved in those references, so we shall use them freely. Under the gauge we have chosen in Sect. 3, we show that tetrahedra have the same meaning as in the Chern-Simons gauge theory.

The basic data are represented by a cross of two edges or a vertex at which three edges meet. We define

$$
\begin{aligned}
& B_{j l}\left[\begin{array}{cc}
m & n \\
i & k
\end{array}\right](+)= \\
& B_{j l}\left[\begin{array}{cc}
m & n \\
i & k
\end{array}\right](-)=
\end{aligned}
$$

Here each line is oriented and marked with an index corresponding to a representation space $\mathscr{H}_{i}$. Since these graphs are open, namely we have outer legs, each open region is also marked. Note that in (4.1-3) all lines go downward.

Now the polynomial identities can be written in the topology language: we construct more complicated graphs from the elementary ones in (4.1-3). If two graphs are equivalent under Reidemeister moves, the quantities represented by these two graphs are equal to each other [14]. We have the following rules in constructing more complicated graphs.

Rule 1. All edges are oriented and marked.

Rule 2. All edges go downward.

Rule 3. Each open region is marked.

Rule 4. Write down a $B$ for a cross of two edges according to (4.1-2).

Rule 5. Write down a $F$ for a vertex where three edges meet according to (4.3). Rule 6. Each closed region is unmarked, dictating a sum over all possible marks.

We will not prove the Reidemeister moves here, but refer to [14]. An important example is the Yang-Baxter equations

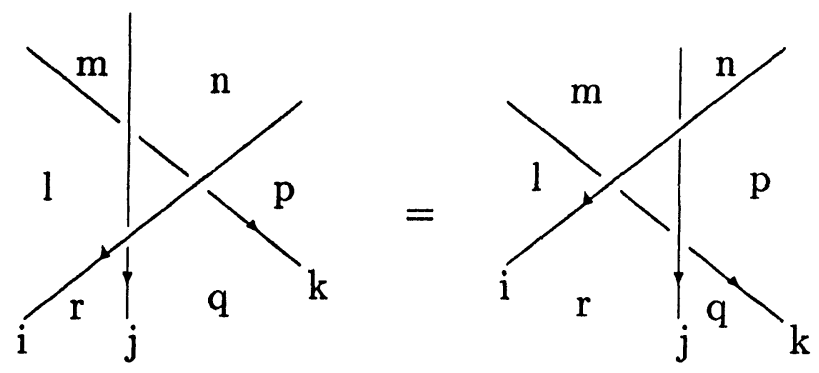


Another example is a kind of pentagon identities
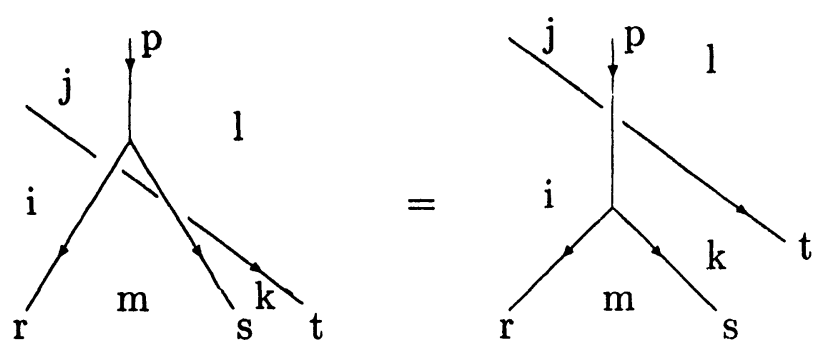

In order to construct more general graphs, in which some edges can go upward or horizontally, we have to define more elementary graphs. Consider

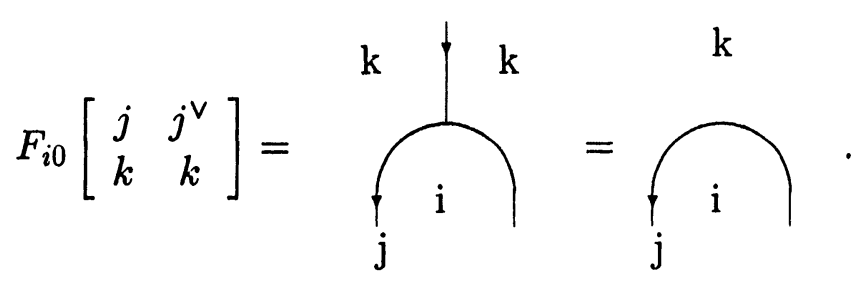

We see from (3.4) that these quantities are not zero. Using the gauge fixed in the last section and Eq. (3.6), we find

$$
F_{i 0}\left[\begin{array}{cc}
j & j^{\vee} \\
k & k
\end{array}\right]=F_{k} / F_{i k}^{j}=F_{j k}^{i} .
$$

We further define new graphs

$$
\left(F_{j k}^{i}\right)^{-1}=\underbrace{\mathrm{j}} \mathrm{k}
$$

and

$$
\mathrm{k} \mid \mathrm{i}=1 \quad \text { if } N_{j i}^{k}>0
$$

Use (4.6), (4.7), and (4.9),

$$
F_{j k}^{i}\left(F_{j k}^{i}\right)^{-1}=\overbrace{\mathrm{j}}^{\mathrm{k}}=\left.\mathrm{k}\right|^{\mathrm{i}} .
$$


By adding (4.8) in the construction of graphs, we can eliminate rule 2. Again Reidemeister moves are guaranteed. For example

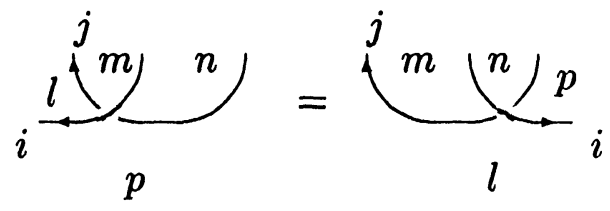

as shown in [14], and

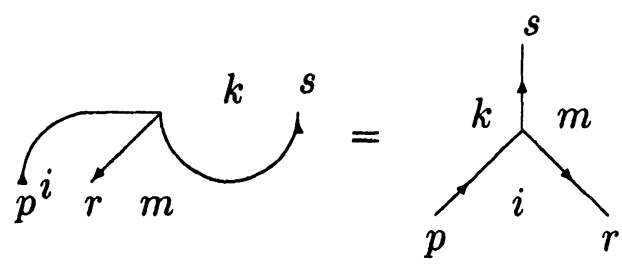

The above equation is equivalent to

$$
F_{m p}\left[\begin{array}{ll}
r & s \\
i & k
\end{array}\right] F_{i 0}\left[\begin{array}{cc}
p^{\vee} & p \\
k & k
\end{array}\right]=F_{i s}\left[\begin{array}{cc}
p^{\vee} & r \\
k & m
\end{array}\right] F_{m 0}\left[\begin{array}{cc}
s^{\vee} & s \\
k & k
\end{array}\right] .
$$

a special case of the pentagon identity.

By (4.8), we can construct closed graphs. The simplest one is

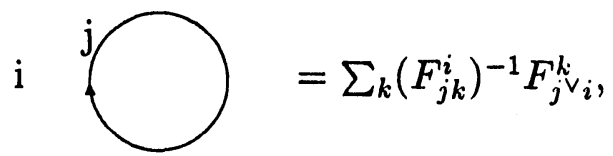

which is related to the element of modular transformation of the characters, and will be discussed in Sect. 6 .

It is straightforward to construct a tetrahedron from the vertices in (4.3). Our first step is

$$
\int_{i}^{r s} k=F_{m p}\left[\begin{array}{cc}
r & s \\
i & k
\end{array}\right] F_{0 r}\left[\begin{array}{cc}
i & m^{\vee} \\
i & m
\end{array}\right] F_{0 s}\left[\begin{array}{cc}
m & k^{\vee} \\
m & k
\end{array}\right] / F_{m}
$$

where we have used (2.8). The second step

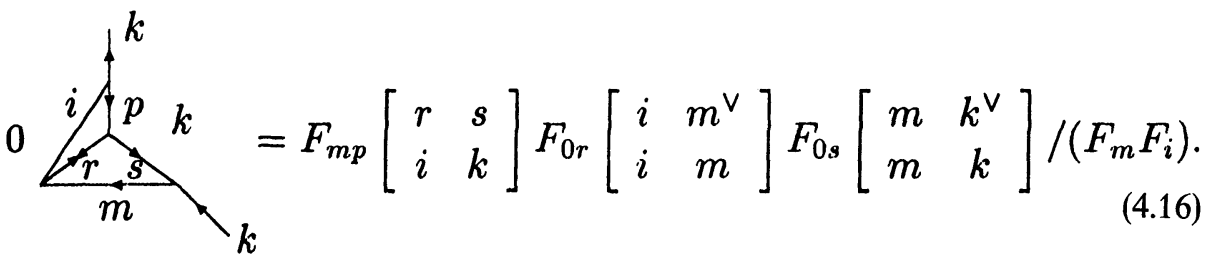


Finally

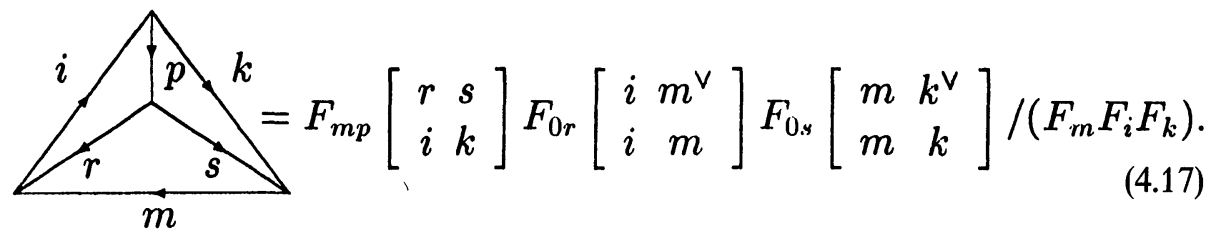

Where we have omitted the mark 0 for the outer region, we shall always do so. Choosing the gauge (3.10) and (3.18), we have a symmetric expression for $F$ in terms of this tetrahedron

$$
F_{m p}\left[\begin{array}{cc}
r & s \\
i & k
\end{array}\right]=\left(F_{i} F_{k} F_{r} F_{s}\right)^{\frac{1}{2}}
$$

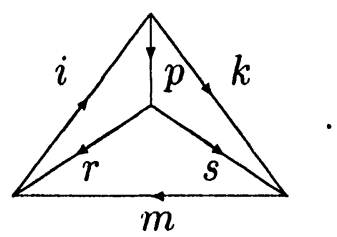

This expression is of the same form as the one by Witten defined in his original paper on the Chern-Simons theory [7]. Here we have no factor $\left(S_{0,0}\right)^{-1} \cdot S_{0,0}$ is the vacuum functional of CS theory. This means that our graphs defined here correspond to the normalized ones in the Chern-Simons theory. We stress that our construction in (4.18) does not invoke any notion of a three dimensional quantum field theory.

\subsection{Tetrahedron Symmetry}

In the Chern-Simons theory, since the tetrahedron diagrams are defined by expectation values of geometric objects [7], the tetrahedron symmetry is an automatic consequence. In the last subsection, however, we defined the tetrahedron more formally for a RCFT. Hence we have to prove its symmetry. The tetrahedron symmetry is a consequence of Reidemeister moves and the following two braiding rules:

and
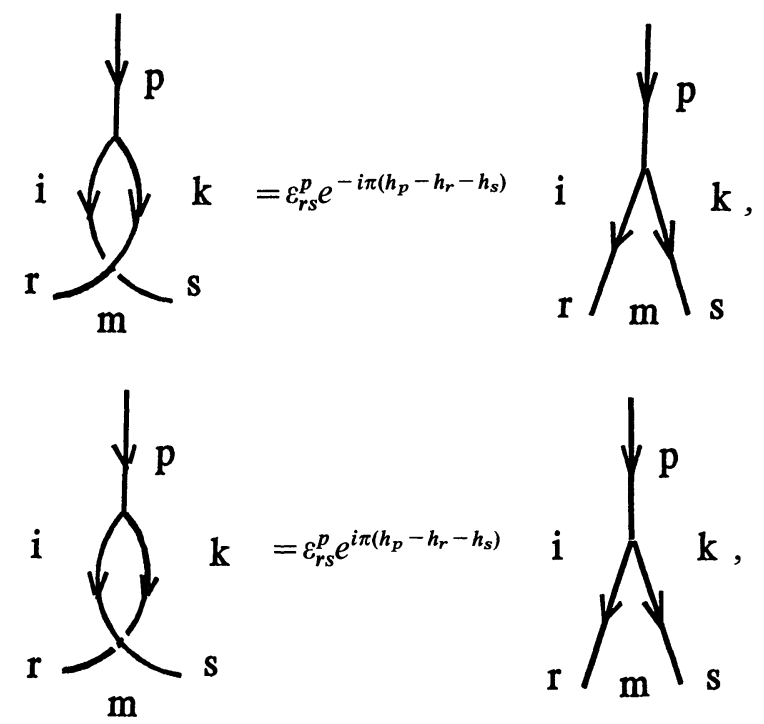
where $\varepsilon_{r s}^{p}$ is a sign depending on the coupling type of the corresponding chiral vertex, $h_{r}$ is the conformal dimension of the highest vector in $\mathscr{H}_{r}$. Equations (4.19-20) are implied by the three point conformal blocks. Before showing the tetrahedron symmetry, we provide a proposition concerning signs appearing in (4.19) and (4.20).

Proposition 1. a) If $N_{i l}^{m}=N_{j m}^{k}=N_{j l}^{n}=N_{n l}^{k}=1$ and

$$
F_{m n}\left[\begin{array}{ll}
j & l \\
k & i
\end{array}\right] \neq 0
$$

then $\varepsilon_{i \varepsilon_{j m}^{m}}^{k}=\varepsilon_{j i}^{n} \varepsilon_{n i}^{k}$.

b) $\varepsilon_{i j}^{m} \varepsilon_{j m}^{j}=\varepsilon_{j j^{\vee}}^{0}$.

c) If $N_{i j k}=1$, then $\varepsilon_{i i^{\vee}}^{0} \varepsilon_{j j^{\vee}}^{0} \varepsilon_{k k^{\vee}}^{0}=1$.

Proof. a) b), and c) follow from a), so we first prove a). Consider the fusing relation

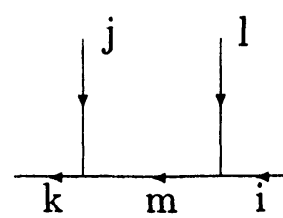

$$
=\sum F_{m n}\left[\begin{array}{ll}
j & l \\
k & i
\end{array}\right]
$$

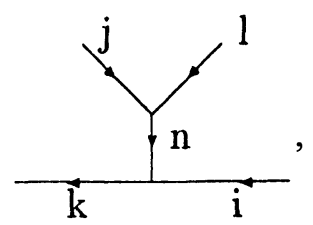

Similarly
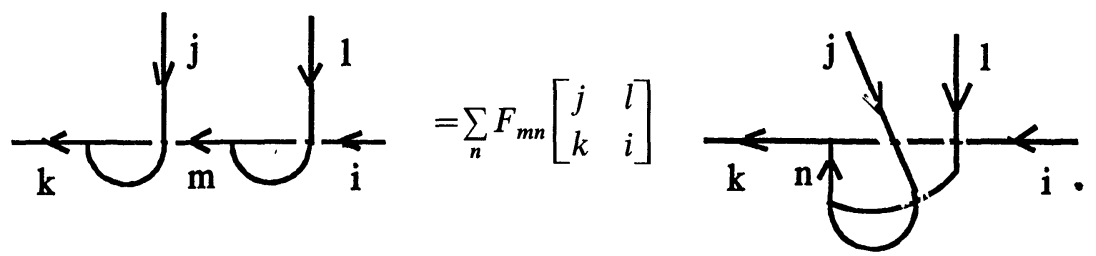

Resolving braidings in the above equation, we find

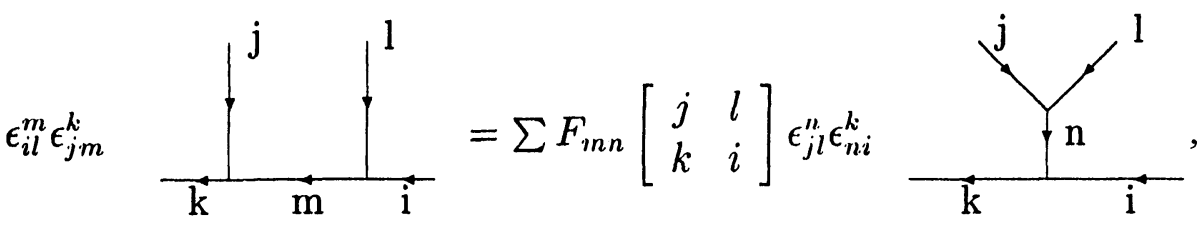

where we have dropped out a common factor associated to conformal weights. Comparing (4.21) with (4.22), we find that $\varepsilon_{i l}^{m} \dot{\varepsilon}_{j m}^{k}=\varepsilon_{j l}^{n} \varepsilon_{n i}^{k}$, since each term on the righthand side of (4.22) is independent. However, when the corresponding entry of the fusing matrix is zero, the proposition needs not be valid.

b) Let in a) $i=k, l=j^{\vee}$, and $n=0$, since

$$
F_{m 0}\left[\begin{array}{cc}
j & j^{\vee} \\
i & i
\end{array}\right]=F_{i j}^{m \neq 0},
$$

so $\varepsilon_{i j}^{m} \varepsilon_{j m}^{i}=\varepsilon_{i i}^{0}$.

c) From b)

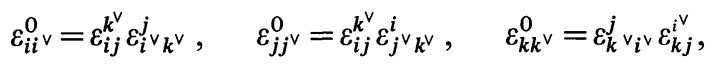

combining these identities, we prove c). 
The term tetrahedron in (4.18) means effectively one can treat it as a tetrahedron in 3 dimensions

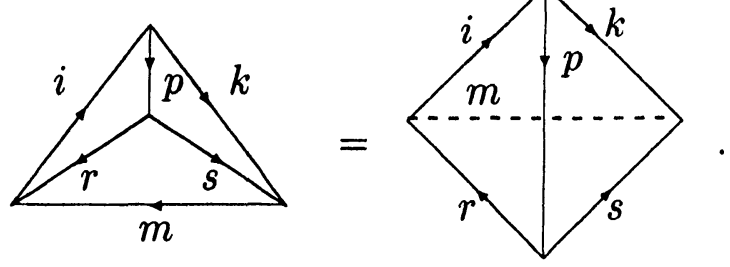

The tetrahedron symmetry in turn implies that we can do various projections of the tetrahedron onto the plane. The resulting various graphs are equal to one another. For example, we have

Theorem 3. There is a tetrahedron symmetry:
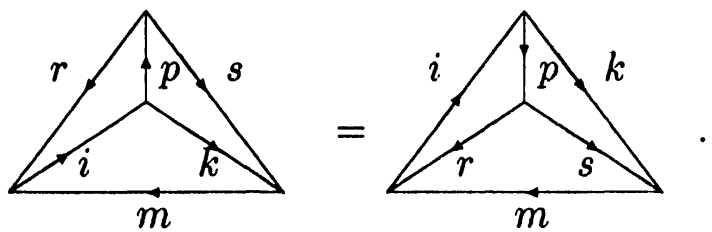

Hence, by virtue of (4.18),

$$
F_{m p^{\vee}}\left[\begin{array}{ll}
i^{\vee} & k \\
r^{\vee} & s
\end{array}\right]=F_{m p}\left[\begin{array}{ll}
r & s \\
i & k
\end{array}\right] .
$$

Proof. We go from the right-hand side of (4.24) to its left-hand side. Using (4.19-20), we have

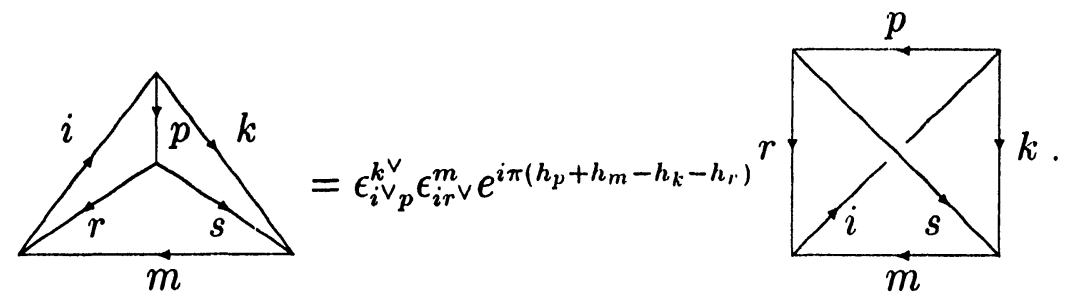

We remark that the graph in the right-hand side of the above equation corresponds to a $B$. Using (4.19-20) again, we achieve
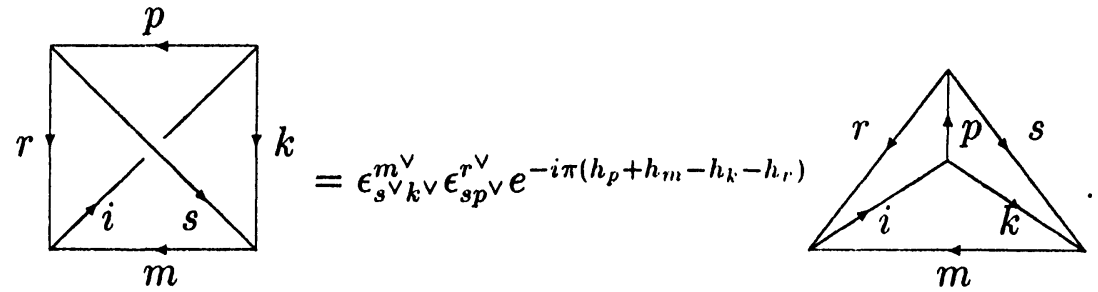
Combining (4.26) and (4.27):
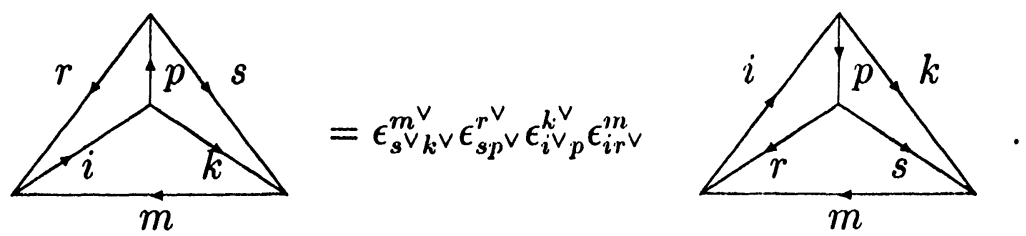

Now applying Proposition 1, whenever the quantities in (4.28) are not zero, we must have

$$
\varepsilon_{s k}^{m} \varepsilon_{s p^{\vee}}^{r^{\vee}} \varepsilon_{i p}^{k^{\vee}} \varepsilon_{i r^{\vee}}^{m}=1
$$

thus we proved (4.24).

\section{Constructing Vector Spaces}

Various operators acting on the conformal blocks and on the states in the Hilbert space have been constructed when one treats a Chern-Simons theory. These operators include generators for the quantum group [11], the half Verlinde operators [13] and the Verlinde operators $[2,5]$. Usually, it is not so easy to work everything out directly in these frameworks. One main issue for the reconstruction problem is to construct vector spaces which are isomorphic to the spaces of conformal blocks from knowing only duality data. To compare with the ChernSimons theory, we shall also define an inner product for the vector space. As we shall see, this can be done for a unitary theory. This fact is not surprising since we know that the rational conformal field theory corresponding to a Chern-Simons theory is unitary.

One purpose for constructing the vector spaces with inner products is that we can express other quantities such as the modular transformations $S(n)$ of one-point blocks in terms of them. In this way, it is straightforward to show that the polynomial identities involving $S(n)$ are not independent of the set of equations given in Sect. 2 [6]. We will provide the proof for $S(n)^{2}=\Theta(n)$ in Sect. 6 .

After constructing all these vector spaces, we have explicit blocks in building a rigid quasitensor category, which we denote as the Moore-Seiberg category (see Appendix B). To recover the quantum group from this category, we have to construct other vector spaces on which the quantum group acts. Mathematically, we have to construct a functor from the Moore-Seiberg category to the category of vector spaces.

We shall assume in this section that our theory is unitary. By the CPT theorem, the F's must satisfy [17]

$$
\bar{F}_{l n}\left[\begin{array}{cc}
i_{1} & i_{2} \\
j & k
\end{array}\right]_{\alpha \beta}^{\gamma \delta}=F_{l^{\vee} n^{\vee}}\left[\begin{array}{ll}
i_{1}^{\vee} & i_{2}^{\vee} \\
j^{\vee} & k^{\vee}
\end{array}\right]_{\alpha^{\vee} \beta^{\vee}}^{\gamma^{\vee} \delta^{\vee}},
$$

where by $\bar{F}$ we mean the complex conjugate of $F$.

Given a triplet (ijk) and any $p$, if $N_{i k}^{j}>0$, we construct the vector space $W_{i k}^{j}(p)$ of dimension $N_{i k}^{j}$ which consists of states

$$
|\alpha\rangle_{p}=\sum_{m, n, \alpha_{i}} \underbrace{j \alpha_{1} i \alpha_{2} k \alpha_{3}}_{\alpha}
$$


where $\left|m, n, \alpha_{i}\right\rangle$ is an arbitrary orthonormal basis, introduced by hands. The number of them is usually larger than $N_{i k}^{j}$. By the inner product among $\left|m, n, \alpha_{i}\right\rangle$, we associate an inner product among $|\alpha\rangle_{p}$. By (5.1) and the tetrahedron symmetry, it is easy to see that we have

$$
{ }_{p}\langle\alpha|=\overbrace{j \sum_{j} i \alpha_{2} k \alpha_{3}}^{\alpha^{v^{p}}}\left(F_{i} F_{j} F_{k}\right)^{-\frac{1}{4}}\left\langle m, n, \alpha_{i}\right| .
$$

The coefficients in the above formula are complex conjugate of those in (5.2). The inner product can be easily calculated

$$
{ }_{p}\langle\alpha \mid \beta\rangle_{p}=j \underbrace{\alpha^{\vee}}_{\beta} k
$$

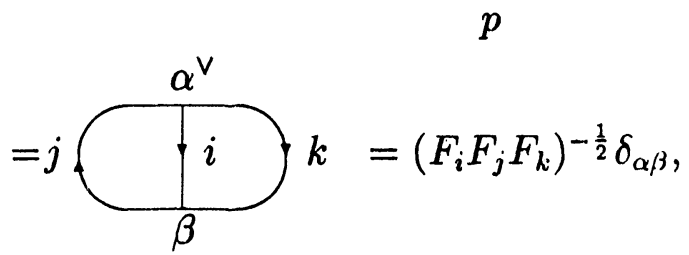

the equality between the first line and the second line can be proved by lassoing the graph in the second line by a cycle marked $p$. We see that all spaces $W_{i k}^{j}(p)$ are isomorphic to each other for fixed (ijk). Since there are $N_{i k}^{j}$ of $\alpha$ and $\left(|\alpha\rangle_{p}\right)$ are orthogonal, we have proved

Theorem 4. The vector space $W_{i k}^{j}(p)$ generated by $\left(|\alpha\rangle_{p}\right)$ is $N_{i k}^{j}$ dimensional.

Denote the total number of vectors $\left|m, n, \alpha_{i}\right\rangle$ by $N_{i k}^{j}(p)$. Since all $|\alpha\rangle_{p}$ are generated by these vectors, there must be $N_{i k}^{j}(p) \geqq N_{i k}^{j}$. This is implied by the above theorem. However we would like to present another proof for this. From (5.2), we learn

$$
N_{i k}^{j}(p)=\sum_{m, n} N_{k p}^{n} N_{i n}^{m} N_{j^{\vee} m}^{p}=\left(N_{j^{\vee}} N_{i} N_{k}\right)_{p}^{p} .
$$

where we defined matrix $\left(N_{i}\right)_{k}^{j}=N_{i k}^{j}$ et al. These matrices satisfy the fusion algebra

$$
N_{i} N_{k}=\sum_{0} N_{i k}^{l} N_{l}
$$

Substitute (5.6) into (5.5), we find

Thus, we proved

$$
\begin{aligned}
N_{i k}^{j}(p) & =\sum_{l} N_{i k}^{l}\left(N_{j^{\vee}} N_{l}\right)_{p}^{p} \geqq N_{i k}^{j}\left(N_{j^{\vee}} N_{j}\right)_{p}^{p} \\
& =N_{i k}^{j} \sum_{l} N_{j^{\vee}{ }_{j}}^{l} N_{l p}^{p} \geqq N_{i k}^{j} N_{j^{\vee} j}^{0} N_{0 p}^{p}=N_{i k}^{j} .
\end{aligned}
$$

Proposition 2. $N_{i k}^{j}(p) \geqq N_{i k}^{j}$.

Define matrix $N_{i}(p)$ by $\left(N_{i}(p)\right)_{k}^{j}=N_{i k}^{j}(p)$, we shall prove 
Proposition 3. Matrices $N_{i}(p)$ form the commutative "fusion algebra"

$$
N_{i}(p) N_{j}(p)=\sum_{l} N_{i j}^{l}(p) N_{l}(p)
$$

Proof. Using the Verlinde formula

$$
N_{i j}^{l}=\sum_{k} \frac{S_{k i} S_{k j} \bar{S}_{k l}}{S_{0 l}}
$$

we diagonalize $N_{i}(p)$ simultaneously by $S$ :

$$
S N_{i}(p) S^{+}=\operatorname{diag}\left(\frac{S_{i l} S_{p l}^{2}}{S_{0 l}^{3}}\right) .
$$

It is straightforward to prove (5.7) by using these diagonal matrices.

Remark. Note that usually

$$
N_{i j}^{0}(p)=\left(N_{i} N_{j}\right)_{p}^{p} \neq C_{i j} \quad \text { and } \quad N_{0 i}^{j}(p)=\left(N_{i} N_{j} \vee{ }_{p}^{p} \neq \delta_{i j} .\right.
$$

$N_{i k}^{j}(p)$ cannot be fusion rules of a rational conformal field theory. Thus, the vector space generated by vectors $\left|m, n, \alpha_{i}\right\rangle$ has no direct physical meaning. At present, we do not know what is the use of the seemingly interesting formula (5.7).

The construction for $W_{i k}^{j}(p)$ can be extended to more complicated cases. For example, the vector space $W_{i_{1} i_{2 k} k}^{j}(p)$ consists of states

$$
|l, \alpha, \beta\rangle=\underbrace{j \alpha_{1} i_{1} \alpha_{2} i_{2} \alpha_{3} i_{3} \alpha_{4}}_{\alpha l \beta}|m|_{p}^{\left.m\right|^{\prime} q}\rangle^{2}, n, q, \alpha_{i}\rangle .
$$

Now it is easy to prove that all these vector spaces are isomorphic to the corresponding spaces of conformal blocks, since we can define the braiding and fusing naturally. The braiding relation and the fusing relation result from polynomial equations, for example
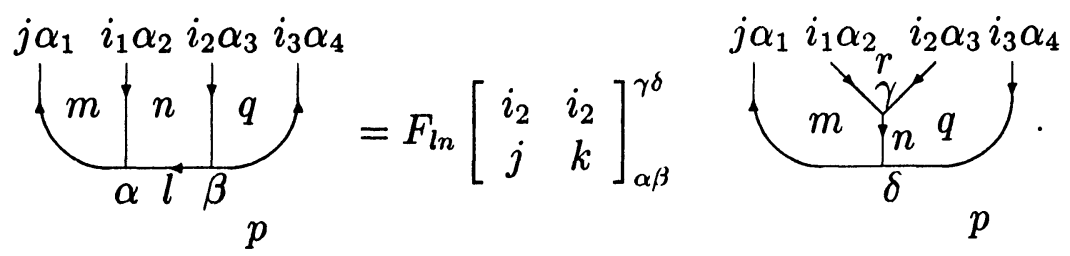

To prove that the above equation is equivalent to the pentagon identity, we need the tetrahedron symmetry to rearrange the indices.

By the notion of the vector spaces, we can express various graphs in terms of the fundamental ones. For example, let

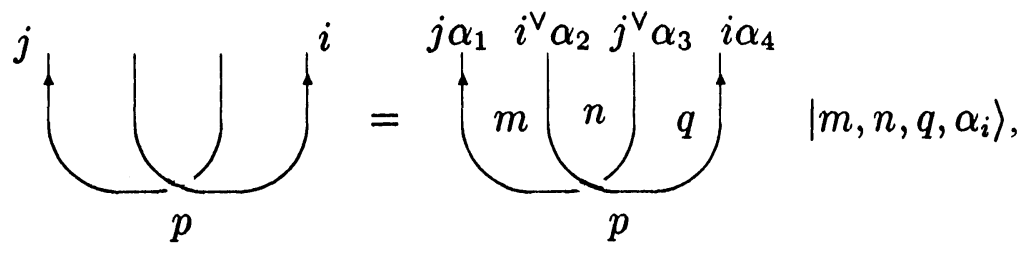


We have

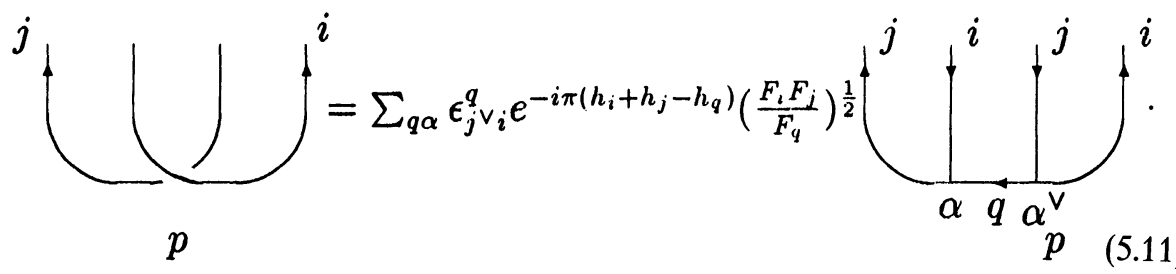

This can be proved by taking the inner product of the vector in (5.11) with the base vectors and checking that the norms of the vectors in the two sides of the above equation are same.

So far we have constructed various vector spaces which are isomorphic to the corresponding spaces of conformal blocks. But for the latter we cannot define the inner product. Recall that in the Chern-Simons theory, the Hilbert spaces have the natural inner product, so our vector spaces defined here are the algebraic reminiscences of the Hilbert spaces, if the correspondence between the RCFT and the Chern-Simons theory exists.

Finally, we discuss the fusing of products of states. Let $\alpha \in W_{i m}^{l}$ and $\beta \in W_{j n}^{m}$, we can define the formal decomposition

$$
|\alpha\rangle \otimes|\beta\rangle=\sum_{\substack{k,|\gamma\rangle \in W_{i j}^{k} \\
|\delta\rangle \in W_{k n}^{l}}} F_{m k}\left[\begin{array}{ll}
i & j \\
l & n
\end{array}\right]_{\alpha \beta}^{\gamma \delta}|\gamma\rangle \otimes|\delta\rangle
$$

which preserves the inner product. To show this let us recall

$$
\left(\left\langle\alpha^{\prime}\left|\otimes\left\langle\beta^{\prime}|,| \alpha\right\rangle \otimes\right| \beta\right\rangle\right)=\delta_{\alpha \alpha^{\prime}} \delta_{\beta \beta^{\prime}}\left(F_{i} F_{j} F_{l} F_{n} F_{m}^{2}\right)^{-1 / 2},
$$

and a similar formula for the inner product of the states appear in the right-hand side of (5.12), and

$$
\bar{F}_{m k}\left[\begin{array}{ll}
i & j \\
l & n
\end{array}\right]_{\alpha \beta}^{\gamma \delta} \stackrel{\mathrm{CPT}}{=} F_{m^{\vee} k^{\vee}}\left[\begin{array}{ll}
i^{\vee} & j^{\vee} \\
l^{\vee} & n^{\vee}
\end{array}\right]_{\alpha^{\vee} \beta^{\vee}}^{\gamma^{\vee} \delta^{\vee}} \stackrel{T . S .}{=} F_{k m}\left[\begin{array}{ll}
l^{\vee} & i \\
n^{\vee} & j
\end{array}\right]_{\delta \gamma}^{\alpha \beta}=F_{k m}^{-1}\left[\begin{array}{ll}
i & j \\
l & n
\end{array}\right]_{\gamma \delta}^{\alpha \beta} .
$$

In the discussion about the quantum group we shall introduce a "restricted" tensor product, in this sense $|\gamma\rangle$ together $F$ in (5.12) may be viewed as an intertwiner. Formally, we can write down

$$
|\alpha\rangle \otimes|\beta\rangle=\sum_{\substack{k, \gamma \\
|\delta\rangle \in W_{k n}^{l}}} F_{m k}\left[\begin{array}{ll}
i & j \\
l & n
\end{array}\right]_{\alpha \beta}^{\gamma \delta}\left(F_{i} F_{j} F_{k}\right)^{-1 / 4}|\delta\rangle_{\gamma},
$$

where the subscript $\gamma$ for $|\delta\rangle$ means that the state $|\delta\rangle$ is in the $\gamma$-th copy of $W_{k n}^{l}$. The factor $\left(F_{i} F_{j} F_{k}\right)^{-1 / 4}$ takes care of the preservation of the inner product. The quantities appearing in the right-hand side in front of $|\delta\rangle_{\gamma}$ serve as the intertwiner for certain operators defined in Sect. 8. But remember that $\left(F_{i} F_{j} F_{k}\right)^{-1 / 4}$ plays no role in the intertwiner, because it is a common factor. We should point out that inspired by (5.9), which is equivalent to the pentagon identity, one can define some variations of (5.15) [21]. This could prove helpful in studying the quantum group.

In conclusion, we have constructed various vector spaces in which a vector can be represented by a graph in which all regions are not marked except for the outer 
one. Inner product of two vectors then is represented by a closed graph, i.e., a graph without outer legs. We can define fusion maps among tensor products of spaces $W_{i k}^{j}$. These maps will be used in discussing the quantum group in Sect. 8.

\section{Modular Covariance for Conformal Blocks on the Torus}

In this section we shall demonstrate how one can prove the polynomial equations involving modular transformations $S(n)$ for one-point blocks on the torus. Moore and Seiberg proved that if the duality properties for the four-point conformal blocks on the sphere and the modular covariance for the one-point blocks on the torus are satisfied by the duality data, the remaining duality properties and the modular covariance of conformal blocks on a Riemann surface of arbitrary genus are guaranteed. We already summarized the polynomial equations for the fusing matrices and the braiding matrices in Sect. 2. These equations are sufficient for constructing topological invariant graphs, as we showed in Sect. 4. In this section, we use the definition for $S(n)$ in Sect. 2 and manipulations on graphs to show that the equations for $S(n)$ are not independent [6]. We only demonstrate the proof for one of them. For the proof of remaining identities, please see [6]. Actually, Proposition 4 and Theorem 5 were proved by the present author and $\mathrm{Yu}$ in [6]. We offer Proposition 5 here in order to show that actually $S(n)$ together $T(n)$ form a projective representation of the modular group of genus one.

For simplicity, we assume that $N_{j k}^{i} \leqq 1$. We write down the definition M2 in Sect. 2 for $S(j)$ again:

$$
\begin{aligned}
S(n)_{i, j} & =\sum_{q} e^{i 2 \pi\left(h_{q}-h_{i}-h_{j}\right)} S_{0, q}\left(T_{q}(n)\right)_{i, j}, \\
\left(T_{q}(n)\right)_{i, j} & =F_{j i}\left[\begin{array}{ll}
n & i \\
j & q
\end{array}\right] .
\end{aligned}
$$

$S_{i, j}$ are entries of the modular transformation for the characters on the torus ${ }^{2}$, corresponding to $\tau \rightarrow-1 / \tau$.

Proposition 4. The above formula has a simple pictorial representation

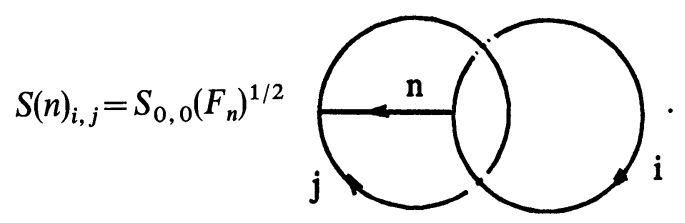

Using the vector spaces constructed in Sect. 5, it is not hard to prove (6.2). In (6.2) we have used the famous identity $[3,17,15] F_{n}=S_{0,0} / S_{0, n},(5.11)$ was also used. Note that (4.14) is a special case of formula (6.2). By (6.2) we are able to prove that $S(n)$ is a unitary matrix and satisfies the first identity $S(n)^{2}=\Theta(n)$ required by modular covariance. Here we define the matrix $\Theta(n)$ as

$$
\Theta(n)_{i, j}=\varepsilon_{i i^{\vee}}^{n} \varepsilon_{i i^{\vee}}^{0} e^{-i \pi h_{n}} C_{i, j} .
$$

where $C$ is the charge conjugation matrix, $C_{i j}=\delta_{i j^{\vee}}$.

${ }^{2}$ It should be interesting to interpret Eq. (6.1) in terms of the quantum group. We will do so in [21] 
$S(n)$ together with $T(n)$, the Dehn twist along the $a$ circle, provides a projective representation of the modular group. If $S(n)$ is block diagonal, then each block corresponds to an irreducible representation. Therefore, if $i$ and $j$ are in the same block, we expect that the phase in the front of $C$ must be same, namely, $\varepsilon_{i i^{\vee}}^{n} \varepsilon_{i i^{\vee}}^{0}$ $=\varepsilon_{j j^{\vee}}^{n} \varepsilon_{j j^{\vee}}^{0}$. This is guaranteed by the following proposition.

Proposition 5. If there exists a $q$ such that $F_{i j}\left[\begin{array}{cc}n & i \\ j & q\end{array}\right] \neq 0$, then $\varepsilon_{i i^{\vee}}^{n} \varepsilon_{i i^{\vee}}^{0}=\varepsilon_{j j^{\vee}}^{n} \varepsilon_{j j^{\vee}}^{0}$.

Proof. Using statement a) in Proposition 1, we find $\varepsilon_{i q}^{j} \varepsilon_{n j}^{j}=\varepsilon_{n i}^{i} \varepsilon_{i q}^{j}$, namely $\varepsilon_{n j}^{j}=\varepsilon_{n i}^{i}$. Next, since $N_{i i^{\vee}}^{n}$ and $N_{j j^{\vee}}^{n}$ are not zero, by b) in Proposition 1, we have $\varepsilon_{i i}^{0}=\varepsilon_{n i}^{i} \varepsilon_{i i^{\vee}}^{n}$ and $\varepsilon_{j j^{\vee}}^{0}=\varepsilon_{n j}^{j} \varepsilon_{j j^{\vee}}^{n}$. Combining identities we obtained, we prove the proposition.

Now if $S(n)_{i, j} \neq 0$, there exists at least one $q$ fulfilling the condition in the above proposition, so the corresponding signs in the front of $C$ in (6.3) agree.

At last, we come to prove

Theorem 5. $S(n)$ is a unitary matrix and satisfies $S(n)^{2}=\Theta(n)$. This implies that $S(n)$ commutes with $\Theta(n)$.

Proof. First we note that the inverse of $S(n)$ is

$$
S^{-1}(n)_{i, j}=\sum_{q} e^{i 2 \pi\left(h_{i}+h_{j}-h_{q}\right)} S_{0, q}\left(T_{q}(n)\right)_{i, j}
$$

It is easy to prove that $S(n) S^{-1}(n)=1$, so that $S(n)$ is actually invertible. To verify this, one notes that matrices $T_{q}(n)$ satisfy the fusion algebra

$$
T_{q}(n) T_{q^{\prime}}(n)=\sum_{m} N_{q q^{\prime}}^{m} T_{m}(n),
$$

as it can be verified from the pentagon identities [16]. In verifying that $S^{-1}(n)$ is the inverse of $S(n)$, we need also the identity $(S T)^{3}=S^{2}$ for the modular transformations (of the characters) on the torus. We shall show later in this section that this equation holds provided

$$
\left(S T S^{-1}\right)_{0 j}=\left(T^{-1} S^{-1} T^{-1}\right)_{0, j}
$$

Similar to (6.2), one can prove the following

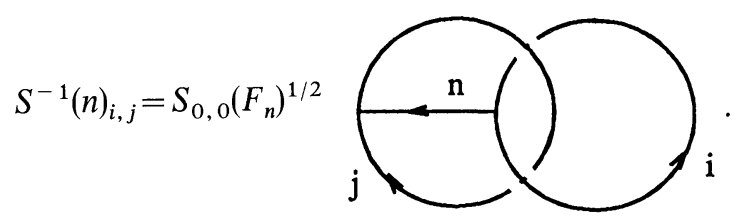

Using the tetrahedron symmetry, we can prove

$$
T_{q}^{T}(n)=T_{q^{\vee}}\left(n^{\vee}\right), \quad T_{q}^{*}(n)=T_{q}\left(n^{\vee}\right),
$$

Subsequently we have $T_{q}^{\dagger}(n)=T_{q^{\vee}}(n)$. Substituting this into $S^{\dagger}(n)$ and noting that $S_{0, q^{\vee}}=S_{0, q}$ we find $S^{\dagger}(n)=S^{-1}(n)$, namely $S(n)$ is a unitary matrix. 
We are ready to prove $S(n)^{2}=\Theta(n)$. Consider the graphic representation of $S^{-1}(n)$ in (6.6). Rotating the right cycle labeled by the index $i$ (rotation can be achieved by simple moves), we find

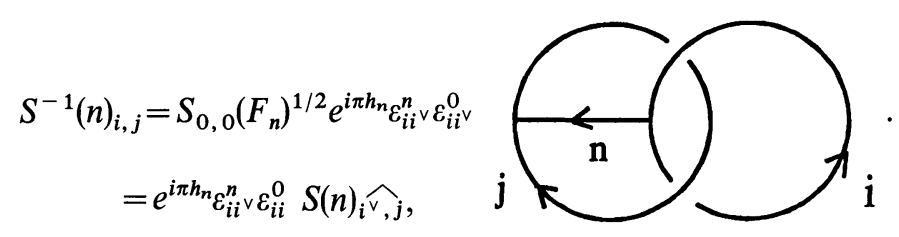

this means $S^{-1}(n)=\Theta^{-1}(n) S(n)$, and $S(n)^{2}=\Theta(n)$. This also implies that $S^{-1}(n)$ $=S(n) \Theta^{-1}(n)$, namely $S(n)$ commutes with $\Theta(n)$. More generally, one can prove that $T_{q}(n) \Theta(n)=\Theta(n) T_{q v}(n)$. This is a consequence of the fact that $T_{q}(n)$ can be diagonalized by $S(n)$, as showed in [6].

Other equations can be proved also by manipulating graphs [6]. Besides the duality property, we need use only $(S T)^{3}=S^{2}$ which is ensured by (6.5), as we shall show shortly. It is a natural conjecture that (6.5) is satisfied by any quantum group.

At last, we would like to point out that by similar graph manipulations one can prove the Verlinde conjecture, using the definition for $S$ in M1 of Sect. 2 [we need not assuming $(S T)^{3}=S^{2}$ here, unlike in the proof for $\left.S(j)^{2}=\Theta(j)\right]$. A special case of the Verlinde conjecture is $S S^{\dagger}=1$, by the property of the fusion rules. We then have $S^{2}=C$. Further, using definition M1 for $S$, we find

$$
T S T=\sum_{q} e^{i 2 \pi\left(\tilde{h}_{q}+\tilde{h}_{0}\right)} S_{0, q} N_{q}
$$

where $N_{q}$ is the matrix of the fusion rules and $\tilde{h}_{i}=h_{i}-c / 24$. Now substitute the Verlinde formula for the fusion rules into the above equation. We deduce

$$
S^{-1} T S T S=\operatorname{diag}\left(e^{i 2 \pi \tilde{h}_{0}}\left(S T S^{-1}\right)_{0 j} / S_{0, j}\right),
$$

we see that provided (6.5) is valid, the above equation implies that $(S T)^{3}=S^{2}$. So all the mess equations concerning modular transformations are eliminated except for (6.5). This is why we include only (6.5) as item C.3 in our whole system of identities in Sect. 2.

Finally we make some remarks on Eq. (6.5). If we have a hidden quantum group structure which organizes all those duality data. Equation (6.5) has a natural interpretation in terms of the quantum group [10]. Notice that in our algebraic axiom system (cf. the end of Sect. 2), (6.5) is the only equation containing the central charge. For a quantum group, the central charge can be defined via an invariant.

\section{A Solution for the Structure Constants}

In this section we discuss the solution of the polynomial equations for the structure constants for a unitary theory. For a physical rational conformal field theory, we have to combine left and right sectors to obtain physical correlator and field operators. Before writing down the formula for the physical fields, let us recall some results obtained before.

Suppose that we already have the largest chiral algebra. Let $(i)$ be the set of labels of primary fields in the left sector and $(\bar{i})$ the set in the right sector. A result 
obtained by Dijkgraaf and Verlinde and by Moore and Seiberg states that there is one-one map $\Pi$ from $(i)$ to $(\bar{i})$. Sometimes we will denote $\bar{i}=\Pi(i)$, such that the physical Hilbert space is

$$
\mathscr{H}=\oplus_{i, \bar{i}} \mathscr{H}_{i} \otimes \overline{\mathscr{H}}_{\bar{i}} ;
$$

remember that 0 is always mapped into $\overline{0}$. The map $\Pi$ is an automorphism of the fusion algebra, namely

$$
N_{i j k}=N_{i \overline{i j k}} \text {. }
$$

In addition, we have $F_{i}=\bar{F}_{i}$.

Bearing this result in mind, we can write down the formula for a physical field

$$
\phi_{\xi_{r} \otimes \bar{\xi}_{\bar{r}}}(z, \bar{z})=\sum_{i, \bar{i}, \bar{j}, \bar{j}, \alpha, \bar{\alpha}} d\left(\begin{array}{c}
i \\
r j
\end{array}\right)^{\alpha \bar{\alpha}} V_{\alpha}^{i j}\left(\left|\xi_{r}\right\rangle, z\right) V_{\alpha}^{\overline{i j}}\left(\mid \bar{\xi}_{\bar{r}}, \bar{z}\right),
$$

where $d$ 's are called the structure constants, they are not the structure constants in the BPZ sense [1].

The polynomial equations originate from the requirements that for three-point correlators and four-point correlators there are no nontrivial monodromies, and the one-point function on the torus must be modular invariant [17]. Moore and Seiberg proved that these structure constants are not zero, provided the corresponding fusion rules are not zero. This is called the naturality theorem [17].

From the three-point correlators, one finds

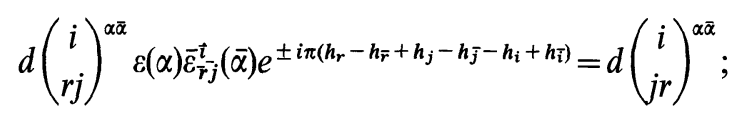

this means that after the braiding, the correlator remains unchanged. There is essentially one phase on the left-hand side of the above equation, since from the two-point correlators we must have

$$
e^{i 2 \pi\left(h_{i}-h_{i}\right)}=1 .
$$

Hereafter we shall use the notation

$$
\tilde{\varepsilon}_{r j}^{i}(\alpha, \bar{\alpha})=\varepsilon_{r j}^{i}(\alpha) \bar{\varepsilon}_{r j}^{\bar{t}}(\bar{\alpha}) e^{ \pm i \pi\left(h_{r}-h_{\bar{r}}+h_{j}-h_{\bar{j}}-h_{i}+h_{\bar{i}}\right)} .
$$

Similar to (7.4), we have

$$
d\left(\begin{array}{c}
i \\
r j
\end{array}\right)^{\alpha \bar{\alpha}} \tilde{\varepsilon}_{v^{\vee}}^{r v}(\alpha, \bar{\alpha})=d\left(\begin{array}{c}
j^{\vee} \\
r i^{\vee}
\end{array}\right)^{\alpha \bar{\alpha}}
$$

Consider the four-point correlators. The following equality

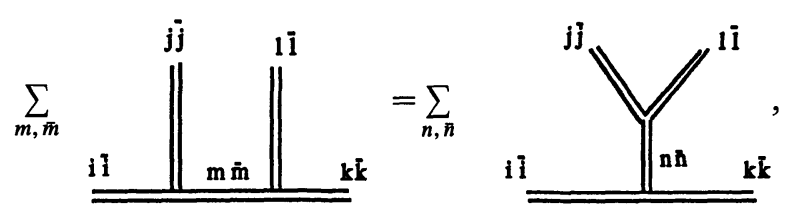

can be understood in two ways. Firstly, by fusing, we obtain the left-hand side from the right-hand side, secondly, both sides must be equal to each other without doing fusing. We thus have

$$
\sum_{m, \bar{m} \alpha \bar{\alpha} \beta, \bar{\beta}} d\left(\begin{array}{c}
i \\
j m
\end{array}\right)^{\alpha \bar{\alpha}} d\left(\begin{array}{l}
m \\
l k
\end{array}\right)^{\beta \bar{\beta}} F_{m n}\left[\begin{array}{cc}
j & l \\
i & k
\end{array}\right]_{\alpha \beta}^{\gamma \delta} \bar{F}_{\bar{m} \bar{n}}\left[\begin{array}{cc}
\bar{j} & T \\
\bar{i} & \bar{k}
\end{array}\right]_{\bar{\alpha} \bar{\beta}}^{\bar{\gamma} \bar{\beta}}=d\left(\begin{array}{c}
n \\
j l
\end{array}\right)^{\gamma \bar{\gamma}} d\left(\begin{array}{c}
i \\
n k
\end{array}\right)^{\delta \bar{\delta}} \delta_{\bar{n}, I(n)},
$$


where all labels with bar are the ones under the map except for $\bar{n}$. This equation is equivalent to

$$
\sum_{\alpha, \beta} d\left(\begin{array}{c}
i \\
j m
\end{array}\right)^{\alpha \bar{\alpha}} d\left(\begin{array}{l}
m \\
l k
\end{array}\right)^{\beta \bar{\beta}} F_{m n}\left[\begin{array}{cc}
j & l \\
i & k
\end{array}\right]_{\alpha \beta}^{\gamma \delta}=\sum_{\bar{\gamma}, \bar{\delta}} d\left(\begin{array}{c}
n \\
j l
\end{array}\right)^{\gamma \bar{\gamma}} d\left(\begin{array}{c}
i \\
n k
\end{array}\right)^{\delta \bar{\delta}} \bar{F}_{\bar{n} \bar{m}^{\vee}}\left[\begin{array}{cc}
\bar{i}^{\vee} & \bar{j} \\
\bar{k}^{\vee} & \bar{l}
\end{array}\right]_{\bar{\delta} \bar{\gamma}}^{\bar{\alpha} \bar{\beta}},
$$

where all the labels with bar are the ones under the map $\Pi$. We have used the fact that

$$
\bar{F}_{\bar{n} \bar{m}}\left[\begin{array}{ll}
\bar{i}^{\vee} & \bar{j} \\
\bar{k}^{\vee} & \bar{l}
\end{array}\right]_{\bar{\delta} \bar{\gamma} \bar{\beta}}^{\bar{\alpha} \bar{\beta}},
$$

as a matrix in indices $\{(\bar{n}, \bar{\alpha}, \bar{\beta}),(\bar{n}, \bar{\delta}, \bar{\gamma})\}$ is the inverse of

$$
\bar{F}_{\bar{m} \bar{n}}\left[\begin{array}{cc}
\bar{j} & \bar{l} \\
\bar{i} & \bar{k}
\end{array}\right]_{\bar{\alpha} \bar{\beta}}^{\bar{\gamma} \bar{\delta}} \text {. }
$$

We can obtain a relation among $d$ 's by considering the braiding of the four-point correlators. This relation is not independent of (7.4), (7.7), and (7.10). So we will investigate only these equations.

Now consider the one-point functions on the torus. The one-point conformal block is represented by the graph

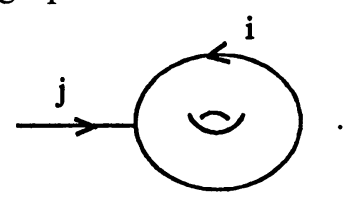

Let $\tau$ be the modular parameter, under the modular transformation $\tau \rightarrow-1 / \tau$ and $\log z \rightarrow \log z / \tau$, the conformal blocks transformed by a matrix $S(j)$. The entry is $S(j)^{l \beta, i \alpha}$. Combining the left sector with the right sector, the modular invariance of the one-point functions implies $[17,3]$

$$
\sum_{i, \bar{i}, \alpha, \bar{\alpha}} d\left(\begin{array}{c}
i \\
j i
\end{array}\right)^{\alpha \bar{\alpha}} S(j)^{l \beta, i \alpha} \bar{S}^{-1}(\bar{j})^{i \bar{\beta}, \bar{i} \bar{\alpha}}=d\left(\begin{array}{c}
l \\
j l
\end{array}\right)^{\beta \bar{\beta}} \overline{\delta_{l, \Pi(l)}},
$$

or equivalently

$$
\sum_{\alpha} d\left(\begin{array}{c}
i \\
j i
\end{array}\right)^{\alpha \bar{\alpha}} S(j)^{l \beta, i \alpha}=\sum_{\bar{\beta}} d\left(\begin{array}{c}
l \\
j l
\end{array}\right)^{\beta \bar{\beta}} \bar{S}(\bar{j})^{\bar{i} \bar{\alpha}, \bar{l} \bar{\beta}}
$$

Note that (7.10) and (7.12) are invariant under the gauge transformation (3.11), provided we scale the $d$ 's simultaneously. After making some reasonable assumption, we will get a solution to (7.4), (7.7), and (7.10). In this case, (7.12) are automatically satisfied. So we doubt if $(7.12)$ are independent of other equations.

Before investigating (7.10), we make the following assumption, which is motivated by Eq. (7.20) and a reason presented after (7.26).

Assumption. Signs defined in (7.6) are independent of $\alpha$ and $\bar{\alpha}$, if $d\left(\begin{array}{c}i \\ r j\end{array}\right)^{\alpha \bar{\alpha}} \neq 0$.

Remark. It is conceivable that the coupling $\alpha$ and $\bar{\alpha}$ is not random. We assume that we have only two cases. The first case is that $\operatorname{even}^{3}$ (odd) $\alpha$ is always coupled to even

${ }^{3}$ By evenness we mean that $\varepsilon_{j m}^{i}(\alpha)=1$, and it is -1 if the coupling is odd 
(odd) $\bar{\alpha}$. The second case is that even (odd) $\alpha$ is always coupled to odd (even) $\bar{\alpha}$. In both cases $\tilde{\varepsilon}_{j m}^{i}(\alpha, \bar{\alpha})$ defined in (7.6) does not depend on $\alpha$ and $\bar{\alpha}$. Currently we are not able to prove this assumption from investigating polynomial equations. It is clear that this assumption is true in case all $N_{i k}^{j} \leqq 1$.

Theorem 6. Under the above assumption and the gauge fixing set in Sect. 3, namely (3.10) and (3.18), we can use the residue gauge transformations satisfying (3.20) to get a diagonal solution to $(7.10)$,

$$
\begin{gathered}
d\left(\begin{array}{c}
i \\
r j
\end{array}\right)^{\alpha \bar{\alpha}}=\delta_{\bar{\alpha}, \Pi(\alpha)}, \quad \tilde{\varepsilon}_{r j}^{i}(\alpha, \bar{\alpha})=1, \\
F_{m n}\left[\begin{array}{cc}
j & l \\
i & k
\end{array}\right]_{\alpha \beta}^{\gamma \delta}=\bar{F}_{\bar{m}^{\vee} \bar{n}^{\vee}}\left[\begin{array}{cc}
\bar{j}^{\vee} & \bar{l}^{\vee} \\
\bar{i}^{\vee} & \bar{k}^{\vee}
\end{array}\right]^{\bar{\gamma}^{\vee} \bar{\delta}^{\vee}}
\end{gathered}
$$

Moreover, (7.12) is automatically satisfied by (7.13).

Proof. We divide our proof into several steps.

Step 1. In (7.10), let $k=\bar{k}=0$, we find

$$
d\left(\begin{array}{c}
i \\
j l
\end{array}\right)^{\gamma \bar{\alpha}} d\left(\begin{array}{c}
l \\
l 0
\end{array}\right)=d\left(\begin{array}{c}
i \\
j l
\end{array}\right)^{\gamma \bar{\alpha}} d\left(\begin{array}{c}
i \\
i 0
\end{array}\right)
$$

where since the entry of $d\left(\begin{array}{c}l \\ l 0\end{array}\right)$ is unique, we neglected the indices. By the naturality theorem, $d$ 's are not always zero in the above equation, so we have

$$
d\left(\begin{array}{c}
l \\
l 0
\end{array}\right)=d\left(\begin{array}{c}
i \\
i 0
\end{array}\right)
$$

if there exists a $j$ such that $N_{j l}^{i}>0$. This is true. Suppose that there is no $j$ coupled to $i$ and $l$, we should have $N^{i} N_{l}=0$, where matrix $N_{i}$, for example, is $\left(N^{i}\right)_{n}^{m}=N_{i n}^{m}$. But let us consider

$$
\left(N^{i} N_{l}\right)_{j^{\vee}}^{i^{\vee}}=\sum_{m} N_{i m}^{i} N_{l l^{\vee}}^{m} \geqq N_{i 0}^{i} N_{l l^{\vee}}^{0}=1,
$$

this contradicts our assumption. So for any pair $i, l,(7.15)$ is valid. This means that $d\left(\begin{array}{c}i \\ i 0\end{array}\right)=$ const. Let us rescale $\phi_{\xi_{i} \otimes \bar{\xi}_{i}}$ such that $d\left(\begin{array}{c}i \\ i 0\end{array}\right)=1$.

Step 2. In (7.10), let $i=\bar{i}=0$, we find

$$
d\left(\begin{array}{c}
0 \\
j j^{\vee}
\end{array}\right) d\left(\begin{array}{c}
j^{\vee} \\
l k
\end{array}\right)^{\gamma \bar{\beta}}=d\left(\begin{array}{c}
0 \\
k^{\vee} k
\end{array}\right) d\left(\begin{array}{c}
k^{\vee} \\
j l
\end{array}\right)^{\gamma \bar{\beta}} .
$$

Using (7.4) and (7.7), the above equation reads

$$
d\left(\begin{array}{c}
0 \\
j j^{\vee}
\end{array}\right) d\left(\begin{array}{c}
j^{\vee} \\
l k
\end{array}\right)^{\gamma \bar{\beta}}=d\left(\begin{array}{c}
0 \\
k^{\vee} k
\end{array}\right) d\left(\begin{array}{l}
j^{\vee} \\
l k
\end{array}\right)^{\gamma \bar{\beta}}
$$

this implies again $d\left(\begin{array}{c}0 \\ i i^{\vee}\end{array}\right)$ is a constant. Let us denote it by $d$.

Step 3. We come to an important case. Let $n=\bar{n}=0$, we have

$$
\sum_{\alpha} d\left(\begin{array}{c}
i \\
j m
\end{array}\right)^{\alpha \bar{\alpha}} d\left(\begin{array}{c}
m \\
j^{\vee}
\end{array}\right)^{\alpha \vee \bar{\beta}} F_{i j}^{m}=d F_{i j}^{\bar{m}} \delta_{\bar{\alpha}, \bar{\beta}^{\vee}}
$$


In our gauge

$$
F_{i j}^{m}=\left(F_{i} F_{j} / F_{m}\right)^{1 / 2}=\left(\bar{F}_{\bar{i}} \bar{F}_{\bar{j}} / \bar{F}_{\bar{m}}\right)^{1 / 2}=\bar{F}_{\bar{i} \bar{j}}^{\bar{m}} .
$$

Now we have to assume that we are treating a unitary theory. By the CPT theorem, we must have [17]

$$
d\left(\begin{array}{c}
i^{\vee} \\
j^{\vee} m^{\vee}
\end{array}\right)^{\alpha^{\vee} \bar{\alpha}^{\vee}}=\overline{d\left(\begin{array}{c}
i \\
j m
\end{array}\right)^{\alpha \bar{\alpha}}}
$$

Combining this condition and the symmetry (7.7), we can cast (7.18) into a simple form

$$
\sum_{\alpha} \tilde{\varepsilon}_{m^{\vee} i}^{j}(\alpha, \bar{\beta}) \overline{\left(\begin{array}{c}
i \\
j m
\end{array}\right)^{\alpha \beta}} d\left(\begin{array}{c}
i \\
j m
\end{array}\right)^{\alpha \bar{\alpha}}=d \delta_{\bar{\alpha}, \bar{\beta}} .
$$

In addition to the above equation, we can obtain various similar equations by considering other simple cases of (7.10). We find they are not independent of the above equation.

Now we use our crucial assumption. We simply drop indices $\alpha$ and $\bar{\beta}$ in the sign $\tilde{\varepsilon}_{m^{v}}^{j}(\alpha, \bar{\beta})$. Equation (7.20) reads in the matrix notation

$$
\tilde{\varepsilon}_{m \vee i}^{j} d\left(\begin{array}{c}
i \\
j m
\end{array}\right)^{\dagger} d\left(\begin{array}{c}
i \\
j m
\end{array}\right)=d \cdot e
$$

where by the superscript $\uparrow$ we mean the hermitian and $e$ is the identity matrix. Since

$$
\operatorname{Det}\left(d\left(\begin{array}{c}
i \\
j m
\end{array}\right)^{\dagger} d\left(\begin{array}{c}
i \\
j m
\end{array}\right)\right)>0
$$

we find $\tilde{\varepsilon}_{m^{\vee}}^{j} d>0$. Consider the special case that $i=0$ and $m=j^{\vee}$ in (7.21), we have $d^{2}=d$, namely $d=1$. Thus

$$
\tilde{\varepsilon}_{m^{\vee}}^{j}=1
$$

Substitute these results into (7.21), we find that $d\left(\begin{array}{c}i \\ j m\end{array}\right)$ is a unitary matrix.

Recall that in Sect. 3, the gauge is not completely fixed. To retain the form of some special $F$ 's in (3.10) and (3.18), which are assumed here, we still have "residual" gauge transformations $A_{j m}^{i}$ satisfying

$$
A_{j m}^{i}=A_{i \vee j}^{m^{\vee}}, \quad\left(A_{i m^{\vee}}^{j}\right)^{T} A_{j m}^{i}=e .
$$

Now we perform a "residual" gauge transformation only in the left sector. Under the gauge transformation, the structure constants transform according to

$$
d\left(\begin{array}{c}
i \\
j m
\end{array}\right)=\left(A_{j m}^{i}\right)^{T} \tilde{d}\left(\begin{array}{c}
i \\
j m
\end{array}\right) .
$$

To preserve the CPT condition (7.19), we must have

$$
A_{i m^{\vee}}^{j}=A_{j^{\vee} m^{\vee}}^{i \vee}=\overline{A_{j m}^{i}} \text {. }
$$

Substituting the above condition to (7.23), we see that $A_{j m}^{i}$ is also a unitary matrix. If we do not want to mix the various coupling types in the space $V_{j m}^{i}$, the matrix $A_{j m}^{i}$ must be block diagonal. Notice that $d\left(\begin{array}{c}i \\ j m\end{array}\right)$ is also block diagonal, by 
our assumption. Since these two matrices are unitary and have the same symmetry [cf. (7.7), (7.22), and (7.23)], we can use $A_{j m}^{i}$ to rescale $d\left(\begin{array}{c}i \\ j m\end{array}\right)$ into a unit matrix. This unit matrix defines a map $\Pi$ from $V_{j m}^{i}$ to $\bar{V}_{\bar{j} \bar{m}}^{i}$. Let us denote $\bar{\alpha}=\Pi(\alpha)$. Feeding back this result to (7.10), we find the condition on the fusing matrices

$$
F_{m n}\left[\begin{array}{ll}
j & l \\
i & k
\end{array}\right]_{\alpha \beta}^{\gamma \delta}=\bar{F}_{\bar{n} \bar{m}}\left[\begin{array}{ll}
\bar{i}^{\vee} & \bar{j} \\
\bar{k}^{\vee} & \bar{l}
\end{array}\right]_{\bar{\delta} \bar{\gamma}}^{\bar{\alpha} \bar{\beta}} .
$$

By the tetrahedron symmetry, we rewrite the above condition as

$$
F_{m n}\left[\begin{array}{ll}
j & l \\
i & k
\end{array}\right]_{\alpha \beta}^{\gamma \delta}=\bar{F}_{\bar{m}^{\vee} \overline{\boldsymbol{n}}^{\vee}}\left[\begin{array}{ll}
\bar{j}^{\vee} & \bar{l}^{\vee} \\
\bar{i}^{\vee} & \bar{k}^{\vee}
\end{array}\right]_{\bar{\alpha}^{\vee} \overline{\boldsymbol{\beta}}^{\vee}}^{\bar{\gamma}^{\vee} \overline{\boldsymbol{\delta}}^{\vee}}
$$

This means that the composite map $\Pi \cdot \vee$ is an automorphism of the fusing matrices. Equation (7.26) is the generalization of the automorphism of the fusing rules. It also generalizes the results in [5], where by some other argument we find $\Pi \cdot \vee$ must be an automorphism among squares of braiding matrices. This independent result suggests that our assumption made before is reasonable.

Using the formula for $S(j)$, we shall show that Eq. (7.12) is automatically satisfied. Because of the gauge invariance, this implies that (7.12) is not an independent equation. Substituting our solution for $d$ 's into (7.12), we obtain a simple equation

$$
S(j)^{l \beta, i \alpha}=\bar{S}(\bar{j})^{\bar{i} \bar{\alpha}, \bar{l} \bar{\beta}} \text {. }
$$

We prove the above equation by going from the right-hand side to the left-hand side. Using the formula for $S(j)$, we proceed

$$
\begin{aligned}
& \bar{S}(\bar{j})^{\bar{i} \bar{\alpha}, \bar{l} \bar{\beta}} \stackrel{\text { def }}{=} \sum_{q, \bar{\gamma}} e^{i 2 \pi\left(h_{q}-h_{i}-h_{l}\right)} \bar{S}_{0, \bar{q}} \bar{F}_{\bar{l} \bar{i}}\left[\begin{array}{ll}
\bar{j} & \bar{i} \\
\bar{l} & \bar{q}
\end{array}\right]_{\bar{\beta} \bar{\gamma}}^{\bar{\alpha} \bar{\gamma}} \\
& \stackrel{\bar{F} \rightarrow F}{=} \sum_{q, \gamma} e^{i 2 \pi\left(h_{q}-h_{i}-h_{l}\right)} S_{0, q} F_{l^{\vee} i^{\vee}}\left[\begin{array}{ll}
j^{\vee} & i^{\vee} \\
l^{\vee} & q^{\vee}
\end{array}\right]_{\beta^{\vee} \gamma^{\vee}}^{\alpha^{\vee} \gamma^{\vee}} \\
& =\sum_{q, \gamma} e^{i 2 \pi\left(h_{q}-h_{i}-h_{l}\right)} S_{0, q} F_{i^{\vee} l^{\vee}}\left[\begin{array}{cc}
j & l^{\vee} \\
i^{\vee} & q
\end{array}\right]_{\alpha \beta}^{\beta \gamma} \stackrel{\text { def }}{=} S(j)^{l^{\vee} \beta, i^{\vee} \alpha},
\end{aligned}
$$

in the last line we used the tetrahedron symmetry. From the last line in the above equation, using again the tetrahedron symmetry, we find

$$
\begin{aligned}
S(j)^{l^{\vee} \beta, i^{\vee} \alpha} & =\sum_{q \vee, \gamma \vee} e^{i 2 \pi\left(h_{q}-h_{i}-h_{l}\right)} S_{0, q} F_{i^{\vee}} l^{\vee}\left[\begin{array}{cc}
j & l^{\vee} \\
i^{\vee} & q^{\vee}
\end{array}\right]_{\alpha \gamma^{\vee}}^{\beta \gamma^{\vee}} \\
& =\sum_{q, \gamma} e^{i 2 \pi\left(h_{q}-h_{i}-h_{l}\right)} S_{0, q} F_{i, l}\left[\begin{array}{cc}
j & l \\
i & q
\end{array}\right]_{\alpha \gamma}^{\beta \gamma}=S(j)^{l \beta, i \alpha} .
\end{aligned}
$$

Finally we proved (7.27), hence (7.12).

Note that $\Theta(j)$ commutes with $S(j)$, so

$$
S(j)^{l \beta, i \alpha}=\left(\Theta(j) S(j) \Theta(j)^{\dagger}\right)^{l \beta, i \alpha}=\varepsilon_{i i^{\vee}}^{0} \varepsilon_{i i^{\vee}}^{j}(\alpha) \varepsilon_{l l^{\vee}}^{0} \varepsilon_{l l^{\vee}}^{j}(\beta) S(j)^{l^{\vee} \beta, i^{\vee} \alpha}
$$

This together with (7.29) implies that if $S(j)^{l \beta, i \alpha} \neq 0$, then

$$
\varepsilon_{i i^{\vee}}^{0} \varepsilon_{i i^{\vee}}^{j}(\alpha) \varepsilon_{l l^{\vee}}^{0} \varepsilon_{l l^{\vee}}^{j}(\beta)=1 .
$$


This is just the statement that an irreducible representation of the modular group extracted from $S(j)$ is indeed a projective representation, since the phase in the corresponding block of $\Theta(j)$ is a constant. We have proved this fact in Proposition 5 in case all $N_{i k}^{j} \leqq 1$.

For a diagonal theory, namely $\Pi=\vee,(7.26)$ hence (7.27) are trivially satisfied. Then we claim that this theory is local and modular invariant, provided we have the duality for both sectors.

Equation (7.13) in Theorem 6 implies that there is an automorphism among all duality data for the left and right sectors. If we believe that duality data determine a quantum group, this in turn implies that the quantum groups originating from the two sectors must be the same. If, a priori the chiral algebras are the same, we automatically have the same quantum groups. Then our results imply that there is a non-trivial automorphism of the quantum group, if the map $\Pi$ is non-diagonal.

Examples. We illustrate our results obtained above by several examples. Our first example is the rational torus model, or rational Gaussian model $[22,17]$. Consider a scalar field compactified on a torus with radius $R^{2}=p / q$, suppose $q$ is an odd integer. The chiral algebra in this case is enlarged $[17,6]$. The primary fields labelled by integers $-2 p q \leqq r \leqq 2 p q$, we have $4 p q$ primary fields. The central charge is 1 and the modular transformations for the characters are

$$
\begin{gathered}
S_{r s}=\frac{1}{\sqrt{4 p q}} e^{i 2 \pi r s / 4 p q}, \\
T_{r s}=\delta_{r s} e^{i 2 \pi\left(r^{2} / 4 p q-1 / 24\right)} .
\end{gathered}
$$

The conjugate of $r$ is $-r$ and the fusion rules are $r \times s=r+s \bmod 4 p q$. In addition to the diagonal model, we have a nondiagonal model in which the map $\Pi$ is given by $2 p s+q r \rightarrow q r-2 p s$. It is easy to check that this map is an automorphism of the fusion rules.

Next we have to check conditions (7.22) and (7.26). Since in our case fusion rules are equal to or less than 1, (7.22) can be obtained without the assumption made about $\bar{\varepsilon}$. First we note that if $s \neq r^{\vee}=-r, s$ and $r$ couple symmetrically [3] (the third field is unique). If $s=r^{\vee}$, we have $\varepsilon_{r r}^{0} \vee=e^{-i \pi r^{2}}$. In both cases we have

$$
\varepsilon_{12}^{3} \varepsilon_{\Pi(1) \Pi(2)}^{\Pi(3)}=1 \text {, }
$$

so we have to check the formula

$$
\exp \left(i \pi\left(h_{1}+h_{2}-h_{3}-h_{\Pi(1)}-h_{\Pi(2)}+h_{\Pi(3)}\right)\right)=1 .
$$

Let the field 1 be labelled by $2 p s_{1}+q r_{1}$ and field 2 by $2 p s_{2}+q r_{2}$. We find

$$
\left(h_{1}+h_{2}-h_{3}\right)-\left(h_{\Pi(1)}+h_{\Pi(2)}-h_{\Pi(3)}\right)=2\left(s_{1} r_{2}+r_{1} s_{2}\right)
$$

which is a even integer, so (7.33) is satisfied. As for condition (7.26), we recall the result obtained in [3] that in a certain gauge it is possible to have all nonvanishing $F$ 's be equal to 1 . This is consistent with our gauge set in Sect. 3. We see that (7.26) is trivially satisfied.

Our second example is the level $k S U(2)$ WZW model. According to the A.D.E. classification [25], we have many nondiagonal theories in this case. We consider $D$-type models first. The modular transformation matrix for $\tau \rightarrow-1 / \tau$ is

$$
S_{i, j}=\sqrt{\frac{2}{k+2}} \sin \frac{(2 i+1)(2 j+1) \pi}{k+2},
$$


where $i, j$ are half integers or integers, it is not hard to see that we have an automorphism of the fusion algebra for $k=4 n+2$ :

$$
\begin{gathered}
\Pi(i)=i \quad i \in Z, \\
\Pi(i)=\frac{k}{2}-i \quad i \in Z+\frac{1}{2} .
\end{gathered}
$$

This corresponds to $D_{2 n-1}$ case. It also corresponds to $S O(3)$ level $4 n+2 \mathrm{WZW}$ model. With the automorphism (7.34), one constructs a modular invariant of the partition function on the torus.

We know that the $\varepsilon$ 's are given by

$$
\varepsilon_{i j}^{l}=(-1)^{i+j-l} \text {. }
$$

Under the map (7.34), we have

$$
\varepsilon_{i j}^{l} \varepsilon_{\Pi(i) \Pi(j)}^{\Pi(l)}=1
$$

Again in this case we must have condition (7.33). But unfortunately (7.33) cannot be always fulfilled. We conclude that we cannot construct a theory with locality with $D_{2 n-1}$ model. This is not a surprise, however. Moore and Seiberg in [23] showed that for $S O(3) \mathrm{WZW}$ model, one has only level $4 n$. This is confirmed in [24] by investigating the Chern-Simons theory. If one introduces the spin structure on the manifold, one can have level $4 n+2$. To construct a local theory with $D_{2 n-1}$, one must allow some twisted fields to be anti-selfconjugate rather than selfconjugate, then one has to modify (7.35). As for $D_{2 n}$ model, the chiral algebra is again extended, this corresponds to $S O(3)$ level $4 n \mathrm{WZW}$ model. Since for this enlarged chiral algebra, the theory is diagonal, we need not to check all those conditions.

Next we consider $E_{7}$ model when $k=16$. We have a nondiagonal modular invariant

$$
Z=\left|\chi_{0}+\chi_{8}\right|^{2}+\left|\chi_{2}+\chi_{6}\right|^{2}+\left|\chi_{3}+\chi_{5}\right|^{2}+\left|\chi_{4}\right|^{2}+\left[\left(\chi_{1}+\chi_{7}\right) \bar{\chi}_{4}+\text { c.c. }\right]
$$

where we use $\chi_{i}$ to denote the character of representation $\mathscr{H}_{i}$ of the Kac-Moody algebra. Obviously here the chiral algebra is enlarged, we have two representations with the same character $\chi_{4}$. Denote these primary fields by $\phi_{4}+$ and $\phi_{4^{-}}$. Denote fields corresponding to $\chi_{0}+\chi_{8}, \chi_{1}+\chi_{7}, \chi_{2}+\chi_{6}$, and $\chi_{3}+\chi_{5}$ by $1, \phi_{1}, \phi_{2}$, and $\phi_{3}$ respectively. The corresponding conformal weights are $0, \frac{1}{9}, \frac{1}{3}, \frac{2}{3}$. The conformal weights of $\phi_{4^{ \pm}}$are $\frac{10}{9}$. From (7.37), we see that there is a nontrivial map $\phi_{1} \leftrightarrow \phi_{4^{+}}$. Dijkgraaf and Verlinde found the fusion rules [2]. Fusion rules which are of interest to us are those involving $\phi_{1}$ and $\phi_{4^{ \pm}}$and the ones larger than 1 . For the latter, we have $N_{33}^{3}=N_{33}^{2}=2$. For the former, we have

$$
N_{111}=N_{112}=N_{122}=N_{123}=N_{133}=N_{134^{+}}=N_{134^{-}}=N_{14^{-4^{+}}}=1 \text {, }
$$

and those under the map $\Pi$. Note that all fields are selfconjugate. We first examine the spaces $V_{33}^{3}$ and $V_{33}^{2}$. It is not hard to see that two couplings in $V_{33}^{3}$ originate from coupling $\mathscr{H}_{3} \otimes \mathscr{H}_{3} \rightarrow \mathscr{H}_{3} \oplus \mathscr{H}_{5}$ and $\mathscr{H}_{3} \otimes \mathscr{H}_{5} \rightarrow \mathscr{H}_{3} \oplus \mathscr{H}_{5}$. The argument for this is the following. Now the chiral algebra is enlarged with the additional current $\phi_{8}(z)$ corresponding to $\chi_{8}$. Under the action this new current the original spaces $\mathscr{H}_{3}$ and $\mathscr{H}_{5}$ are exchanged, due to the old fusion rules. Under the original chiral algebra we have

$$
\left(\mathscr{H}_{3} \oplus \mathscr{H}_{5}\right) \otimes\left(\mathscr{H}_{3} \oplus \mathscr{H}_{5}\right)=4\left(\mathscr{H}_{3} \oplus \mathscr{H}_{5}\right)+\ldots
$$


namely we have four intertwiners. Since under $\phi_{8}(z) \mathscr{H}_{3}$ and $\mathscr{H}_{5}$ are exchanged, only two of the 4 intertwiners survive which are invariant under this new current. These two couplings are antisymmetric. Similar argument applies to $V_{33}^{2}$, in which all couplings are symmetric. We have no additional condition derived in these cases, because the map $\Pi$ is identical here. To check condition (7.22) for nonvanishing fusion rules in (7.38), note that $h_{1}-h_{4^{+}}=-1$ and $(-1)^{1-4}=-1$ when we use Eq. (7.35). The condition can be easily checked to be fulfilled.

\section{Some Remarks on Quantum Groups in RCFT}

It is by now widely believed that the duality properties of a rational conformal field theory are encoded neatly in the representation theory of a quantum group [8]. There have been many discussions on the hidden quantum group in a rational conformal field theory $[10,13,11,15,19]$. For a WZW model, say, of group $S U(N)$, the quantum group is $U_{q}(S U(N))$, where $q$ is a root of unity $\exp \left(\frac{i 2 \pi}{k+N}\right)$. Despite these discussions, a full proof of the existence of a hidden quantum group for a RCFT is still lacking. The work of Alvarez-Gaumé et al. [10] only provides evidence for the WZW model, while the discussion of Moore and Reshetikhin [11] is purely phenomenological. One can also reveal the structure of the quantum group by studying the Knizhnik-Zamolodchikov equations. But in general, we have no corresponding set of differential equations for an arbitrary RCFT. Most recently, Majid [19] gave a formal construction of the quantum group, starting from a rigid quasitensor category (see Appendix B). Still this is not a complete proof, since one must assume existence of a functor from this category to the category of vector spaces which provide the representations of the quantum group. It seems plausible that the final and perhaps most important step is to construct such a functor, which includes the knowledge of the representation spaces and Clebsch-Gordon coefficients. This section is devoted to such a goal. The goal is partly achieved here, in the sense that for those models for which one can perform Witten's lassoing we can obtain the needed CG coefficients. Indeed we also construct the generators for the vertex quantum explicitly. Although we cannot prove that certain limits do exist, we give some evidence for them to exist. Moreover, if such a lassoing prescription indeed works, we show in Appendix B that it actually gives rise to a quantum group.

As the first step, we discuss some aspects of work in [13] in the sense of the quasitriangular Hopf algebra. A few points are clarified. Note that the quasitriangular Hopf algebra discussed in the next subsection is not the one we hope to find.

\subsection{The IRF Quasitriangular Hopf Algebra}

The exact definition of a quasitriangular Hopf algebra (quantum group) is given in Appendix A. In this subsection we shall construct an IRF quantum group for a rational conformal field theory. This quantum group was first introduced in [13]. The term IRF means that the representation matrix of an element has indices of $i$ and so on, which are just used to label the representations of the underlying quantum group which corresponds to the vertex model $[8,12,18]^{4}$. Here we

\footnotetext{
${ }^{4}$ We name it the vertex quantum group. For the WZW model of group $S U(N)$, it is $U_{q}(S U(N))$
} 
introduce various vector spaces $W_{i}$ on which the IRF quantum group acts. These are the prototypes of $V_{i}$ on which the vertex quantum group acts. We also define the antipode and counit and all properties of a quantum group follow. Thus we complete the study in [13].

Alvarez-Gaumé et al. [13] defined a set of operators acting on the following vector space:

$$
W=\oplus_{i, j, k} W_{i k}^{j},
$$

where $W_{i k}^{j}$ is the vector space constructed in Sect. 5 . We have already seen that this space is isomorphic to the space of chiral vertices; in addition we can define an inner product in this case (Sect. 4). Actually, the following vector spaces are invariant under the action of the operators we will define later

$$
\begin{aligned}
& W_{i}=\oplus_{j, k} W_{i k}^{j}, \\
& W=\oplus_{i} W_{i} .
\end{aligned}
$$

As we shall see later, by the Brauer's theorem, the representation space $V_{i}$ of the vertex quantum group is a subspace of $W_{i}$, when we consider the WZW model.

Let $\Phi_{u}$ denote a state in $W$, we write $u=\left(\begin{array}{c}j_{u} \\ i_{u} k_{u}\end{array}\right)_{\alpha_{u}}$ when $\phi_{u} \in W_{i k}^{j}$. The braiding matrices can be written in terms of indices $u, v$ et al.
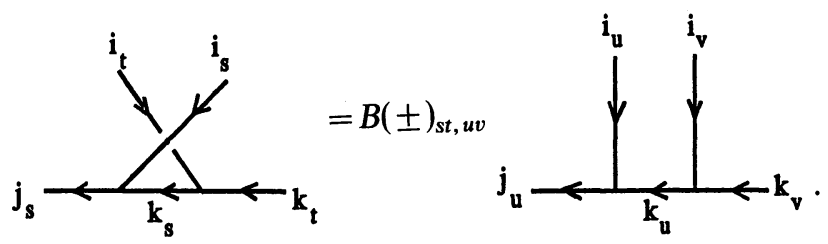

Explicitly, we have

$$
B( \pm)_{s t, u v}=\delta_{k_{s}, j_{t}} \delta_{i_{u}, i_{t}} \delta_{i_{v}, i_{s}} \delta_{k_{v}, k_{t}} \delta_{j_{u}, j_{s}} \delta_{k_{u}, j_{v}} B_{k_{s} k_{u}}\left[\begin{array}{ll}
i_{s} & i_{t} \\
j_{s} & k_{t}
\end{array}\right]_{\alpha_{s} \alpha_{t}}^{\alpha_{u} \alpha_{v}} .
$$

We define two matrices $B( \pm)$ acting on the tensor space $W \otimes W$ by

$$
B( \pm)\left(\phi_{s} \otimes \phi_{t}\right)=\sum_{u, v} B( \pm)_{s t, u v} \phi_{u} \otimes \phi_{v} \text {. }
$$

As maps $B( \pm)$ have no inverse, because many vectors in $W \otimes W$ are sending to zero. Only when they are defined on the "restricted" tensor product space which we will define shortly, they have inverse. For our present purpose, we will not define the restricted tensor product for $W$.

The two Yang-Baxter equations can be easily written in terms of $B( \pm)$. Consider the tensor product space $W \otimes W \otimes W$, let $B_{12}( \pm)$ be the corresponding $B$ 's acting on the first two $W$ 's, and so on. We have the Yang-Baxter equations

$$
B_{12}( \pm) B_{23}( \pm) B_{12}( \pm)=B_{23}( \pm) B_{12}( \pm) B_{23}( \pm)
$$

Let $\tau$ be the twist map: $W \otimes W \rightarrow W \otimes W$, defined by exchange of the order of the tensor product. Define $R( \pm)=\tau B( \pm)$, the above Yang-Baxter equations can be written as

$$
R_{12}( \pm) R_{13}( \pm) R_{23}( \pm)=R_{23}( \pm) R_{13}( \pm) R_{12}( \pm)
$$


Observing these equations, we soon find that if we view $R_{13}( \pm)$ as operator valued matrices with the operator acting on the third $W$ and do so for $R_{23}( \pm)$, we can rewrite the Yang-Baxter equations as operator equations

$$
R( \pm)(T( \pm) \otimes 1)(1 \otimes T( \pm))=(1 \otimes T( \pm))(T( \pm) \otimes 1) R( \pm)
$$

where entries of matrices $T( \pm)$ are $T_{s t}( \pm)$ which are operators acting on $W$ :

$$
T_{s t}( \pm)\left(\phi_{u}\right)=\sum_{v} B( \pm)_{s u, v t} \phi_{v} .
$$

In this way we recover the operators defined in $[13]^{5}$. Before proceeding to check all the properties of a quasitriangular algebra defined by $T_{s t}( \pm)$, we first consider the vector spaces on which these operators act.

The key point we find is new is that $W$ is not the irreducible representation space of the operators $T$, rather, the subspaces $W_{i}$ themselves form possibly irreducible representation spaces of these operators. This is easily seen by the definition (8.9) and the formula (8.4). Namely, under $T_{s t}( \pm)$ a state $\phi_{u}$ in $W_{i}$ is mapped into $W_{i}$. To define the coproduct, we first define the "restricted" tensor product of $W_{i}$ and $W_{j}$. Let $\phi_{u} \in W_{i}$ and $\phi_{v} \in W_{j}$. We define $\phi_{u} \otimes \phi_{v}=\phi_{u} \otimes \phi_{v}$ whenever $k_{u}=j_{v}$ (see the graph on the right-hand side of (8.3)), otherwise it is zero. By fusing, we have

$$
\begin{aligned}
& W_{i} \otimes W_{j}=\left(\oplus_{l, m} W_{i m}^{l}\right) \otimes{ }^{\prime}\left(\oplus_{m^{\prime}, n} W_{j n}^{m^{\prime}}\right) \\
& \quad=\bigoplus_{m, l, n} W_{i m}^{l} \otimes W_{j n}^{m} \stackrel{\mathbb{F}}{\cong} \oplus_{k, l, n} W_{i j}^{k} \otimes W_{k n}^{l}=\oplus_{k} W_{i j}^{k} W_{k} .
\end{aligned}
$$

In the second line we used the map $F$ defined in Sect. 5. Thus we see that the restricted tensor product of two vector spaces $W_{i}$ and $W_{j}$ decomposes into a direct sum of $W$ 's, which is the desired result for the representation theory of a Hopf algebra. To clarify this point, we shall define the coproduct of the algebra $A$ generated by $T_{\text {st }}( \pm)$. The coproduct $\Delta$ is a map from $A$ to $A \otimes A$. We define

$$
\Delta\left(T_{s t}( \pm)\right)=\sum_{u} T_{s u}( \pm) \otimes T_{u t}( \pm) .
$$

To show that this definition of the coproduct is consistent with decomposition of (8.10), namely the elements in $W_{i j}^{k}$ are intertwiners of the representations of the algebra, we have to show that the following diagram is commutative

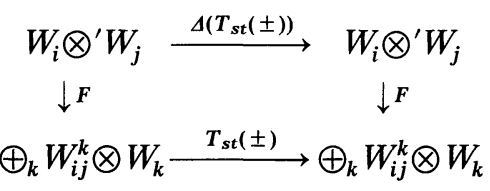

This is guaranteed by the pentagon identity. The above diagram is the refined version of condition (2.19c) in [13] for defining properties of operators T's. By definition, it is straightforward to check the coassociativity $(\Delta \otimes \mathrm{id}) \Delta=(\mathrm{id} \otimes \Delta) \Delta$, where id is the identity map from $A$ to $A$.

The relations (8.8) among $T$ 's are equivalent to the following relations:

$$
\begin{aligned}
& R(+) T_{1}(+) T_{2}(+)=T_{2}(+) T_{1}(+) R(+), \\
& R(+) T_{1}(-) T_{2}(-)=T_{2}(-) T_{1}(-) R(+),
\end{aligned}
$$

\footnotetext{
${ }^{5}$ Note that in [13] only one set of $T_{s t}$ is introduced
} 
where we use $T_{1}\left(T_{2}\right)$ to denote $T \otimes 1(1 \otimes T)$. Note that the first relation is the old one. To prove the second one, we start from the Yang-Baxter equation

$$
R_{31}(-) R_{32}(-) R_{12}(-)=R_{12}(-) R_{32}(-) R_{31}(-) ;
$$

this is just (8.7) with re-enumerated indices. As we mentioned before, $R(-)$ is not invertible. But effectively if we view it acting on the restricted tensor product, it is invertible and we find

$$
R_{12}(+) R_{13}(-) R_{23}(-)=R_{23}(-) R_{13}(-) R_{12}(+),
$$

where we have used $R(+)=\tau R^{-1}(-) \tau$. The above equation is equivalent to the second identity in (8.13). So the two identities in (8.13) are equivalent to the two Yang-Baxter equations. We can derive some other formulas for the $T$ 's, but they are not independent. For example

$$
R(+) T_{1}(+) T_{2}(-)=T_{2}(-) T_{1}(+) R(+) .
$$

We stress that although the above identity is not independent of (8.13) when we know the definition (8.9) of the representations $W_{i}$, it should be an independent defining relation when we treat the algebra $A$ abstractly. Associated with a matrix $R(+)$ and generators $T( \pm)$, we have a Hopf algebra with relations (8.13) and (8.15), provided we define the proper coproduct, the antipode and the counit [18].

To cast the algebra $A$ into a Hopf algebra, we still have to define the antipode $S$ and the counit $\varepsilon$. These are defined by

$$
S\left(T_{s t}( \pm)\right)=T_{t s}(\mp), \quad \varepsilon\left(T_{s t}( \pm)\right)=\delta_{s t} .
$$

The above formulas can be deduced from the consistency conditions (presented in Appendix A) for a Hopf algebra.

We claim that the Hopf algebra $A$ we have defined is a quasitriangular Hopf algebra. First of all, all conditions on the coproduct, the antipode and the counit are satisfied. The universal $R$-matrix can be found in a sophisticated way. Remember that, although superficially what relations ${ }^{6}$ we have checked are the same for the definition of algebra $\breve{U}(R(+))$ defined in, say [18], this algebra is essentially not $\breve{U}(R(+))$. The reason is that $T_{s t}( \pm)$ are not always nonzero for any pair $s, t$. They are not zero only when $i_{s}=i_{t}$, as can be seen from (8.4). The decomposition property (8.10) strongly suggests that besides $W_{i}$, we have no more essentially new representations, since usually all other representations can be generated by taking the tensor products of some "fundamental" representations, as in the representation theory of a group.

\subsection{The Vertex Quantum Group - Witten's Lassoing}

As we mentioned previously, the missing key point in the work of Majid on the reconstruction of the vertex quantum group is the reconstruction of the representation spaces. If, however, we have a family of solutions to the polynomial equations which are parametrized by some discrete parameters, as for the WZW models, we can analytically extend the parameters to the complex domain. In this case, we take infinite limit of some indices in certain quantities and construct the missing ones in the reconstruction of the quantum group. This was first pointed

\footnotetext{
${ }^{6}(8.13)$ and (8.15) together with the definitions of the coproduct, the antipode and counit
} 
out by Witten for the $S U(2) \mathrm{WZW}$ model [12]. We call this procedure the lassoing procedure, since this is based on the observation that the value of a closed graph does not depend on the mark of the outer region so that we can take the infinite limit of this mark.

Now suppose we are dealing with a WZW model. Each representation label $i$ is the highest weight of this representation of the corresponding Lie algebra. Let us consider the triplet ( $i j k)$ when $N_{i k}^{j}>0$. By the Brauer theorem, $j-k$ is a weight in the representation $i$. And in fact $j-k$ together with $\alpha$ corresponds to a vector in this representation, mark this vector by $a$. For simplicity we assume here $N_{i k}^{j}=1$, so we will neglect the index $\alpha$. Note that by the "phenomenological" observation for WZW models, we find the space $W_{i}$ is larger than the representation space $V_{i}$ of the vertex quantum group ${ }^{7}$. The restricted tensor product among the $W_{i}$ 's defined in (8.10) is the analogy of the restricted tensor product among the $V_{i}$ 's defined in [10]. We believe that the IRF quantum group and its representations encode all information for constructing the vertex quantum group. However, at present we have to use the lassoing procedure introduced by Witten in [12]. This method has some drawbacks. First of all, we can provide only some evidence that certain limits exist, we cannot prove that they exist. However at the end of this subsection we demonstrate some examples in which our lassoing procedure works. Second, we do not know whether or not any given RCFT is one in a series of RCFT's.

Our first observation is that we can recover the Clebsch-Gordon coefficients from the $F$ 's lassoing. Suppose $V_{i}$ and $V_{j}$ are the irreducible representation spaces of the quantum group. Let $|i, a\rangle$ be a state in $V_{i}$ and $|j, b\rangle$ a state in $V_{j}$. By the C-G coefficients we can decompose the tensor product of these states into the states in the irreducible representations

$$
|i, a\rangle \otimes|j, b\rangle=\sum_{k c} K_{k c}^{i a, j b}|k, c\rangle,
$$

The weight is conserved, namely we have $c=a+b$. Now the fusing matrix is the map from $V_{i} \otimes\left(V_{j} \otimes V_{k}\right) \rightarrow\left(V_{i} \otimes V_{j}\right) \otimes V_{k}$, we must have

$$
\sum_{d} K_{l e}^{i a, m d} K_{m d}^{j b, k c}=\sum_{n, d} F_{m n}\left[\begin{array}{ll}
i & j \\
l & k
\end{array}\right] K_{n d}^{i a, j b} K_{i e}^{n d, k c} .
$$

If we denote $K$ by the following diagram

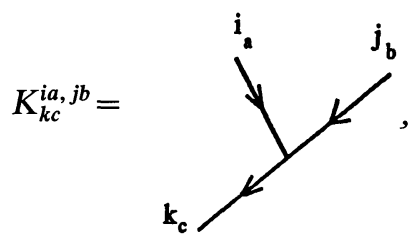

then $(8.18)$ can be written in terms of diagrams

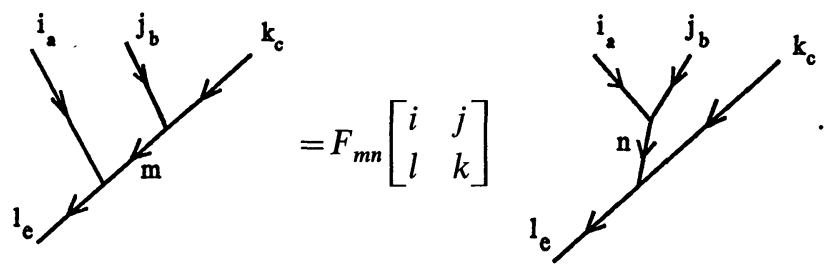

7 The representation $V_{i}$ is the deformation of the corresponding representation of the ordinary group 
We find that this equality is similar to (5.9). In fact let us define

$$
K_{k c}^{i a, j b}=\lim _{p \rightarrow \infty}\left(F_{i s}^{t} F_{j t}^{p} F_{k s}^{p}\right)^{1 / 2} \underbrace{\mathbf{k}}_{\mathrm{p}} \underbrace{\mathrm{i}}
$$

where $a=s-t, b=t-p$, and $c=s-p$. We will argue later that the limit exists. Note that for the fixed triplet (ijk), since $c=a+b$, we have only two independent parameters in the $\mathrm{C}-\mathrm{G}$ coefficient. However in the right-hand side of the above equation, since we have taken limit $p \rightarrow \infty$, we also have two independent parameters. Multiplying an appropriate factor on both sides of (5.9), summing over $s$ and taking the limit $p \rightarrow \infty$, it is not hard to see that (8.18) is a consequence of (5.9). We find that the functor property of $F: V_{i} \otimes\left(V_{j} \otimes V_{k}\right) \rightarrow\left(V_{i} \otimes V_{j}\right) \otimes V_{k}$ follows from the pentagon identity, since (5.9) is equivalent to the pentagon identity.

Now we argue that the limit defined in (8.21) seems to exist. First we consider the following limit

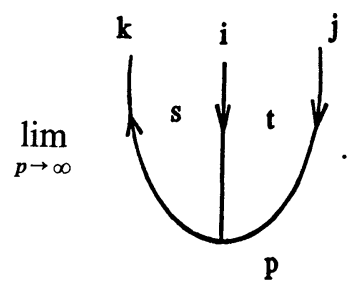

These quantities are components of a vector in the space $W_{i k}^{j}(p)$ defined in the Sect. 5 , so we have

$$
\sum_{r s} \text { square of the absolute value of } \underbrace{\mathbf{r}}_{\mathbf{p}} \underbrace{\mathbf{k}}=\left(F_{i} F_{j} F_{k}\right)^{-1} \text {. }
$$

The above equation implies that the quantities in both sides are positive. After analytically extending the parameter, these quantities become complex. When $p$ is sufficiently large, the numbers of $s$ and of $t$ depend only on ( $i j k)$, as one can see from the fusion rules. So the sum contains finitely many terms. The quantity in the righthand side of the above equation is finite, so each term in the sum is bounded. This suggests that the limit exists except for the ambiguity in the phase. Next we check

$$
\lim _{s \rightarrow \infty} F_{i s}^{t} \text {. }
$$

Since

$$
\sum_{t} F_{i s}^{t}\left(F_{i t}^{s}\right)^{-1}=\sum_{t} F_{s} / F_{t}=F_{i}^{-1}
$$


again the finite sum has a finite value even when $s$ is large; this suggests the limit $\lim F_{s} / F_{t}$ exists, so does $\lim F_{i s}^{t}$. Combining our results, we have argued that the $\stackrel{s \rightarrow \infty}{\text { limit in (8.21) exists. }}$

Thus, by the lassoing procedure, we have defined the maps

and the maps

$$
K: V_{i} \otimes V_{j} \rightarrow V_{k}
$$

$$
F: V_{i} \otimes\left(V_{j} \otimes V_{k}\right\} \rightarrow\left(V_{i} \otimes V_{j}\right) \otimes V_{k}
$$

which are consistent with $K$. These enable us to define the forgetful functor from the Moore-Seiberg category to the category of the vector spaces (see Appendix B). By the formal approach in [19], we can recover the quantum group.

However, without invoking the formal approach in [19], we can define the generators of the quantum group intuitively. As we have pointed out in the end of the last subsection, $i_{s}=i_{t}$ for the operators $T_{s t}( \pm)$ defined previously. So let us consider here only those $T_{s t}( \pm)$ with $i_{s}=i_{t}=i$, here $i$ is fixed. Thus $s$ and $t$ take values in the vector space $W_{i}$. Next we consider the operators $B( \pm)$ acting on $W_{i} \otimes W_{i}$. By the definition, $B( \pm)$ map $W_{i} \otimes W_{i}$ into itself, so do $R( \pm)$. Now (8.8) is valid when restricted to act on $W_{i} \otimes W_{i}$. We also see that the coproduct, antipode and the counit are well-defined when we consider only the operators mentioned above. So we obtain a Hopf algebra generated by these operators.

Let $\phi_{u}$ be an element in $W_{j}$. Consider $T_{s t}( \pm)$ acting on $\phi_{u}$. We rewrite (8.9) here

$$
T_{s t}( \pm)\left(\phi_{u}\right)=\sum_{v} B_{s u, v t} \phi_{v} \text {. }
$$

We take an infinite limit for $k_{s}$ again. All of the following become the infinite

We define

$$
\begin{array}{ll}
k_{s}=j_{u} & j_{s}=j_{v}, \\
j_{t}=k_{v} & k_{t}=k_{u} .
\end{array}
$$

$$
\begin{array}{ll}
j_{s}-k_{s}=a_{s}, & j_{t}-k_{t}=a_{t}, \\
j_{u}-k_{u}=a_{u}, & j_{v}-k_{v}=a_{v} .
\end{array}
$$

These values are finite. Similarly, we can argue that the following limit exists (see also [12])

$$
\lim _{k_{s} \rightarrow \infty} B_{s u, v t} \text {. }
$$

We denote this quantity by $B_{a_{s} a_{u}, a_{v} a_{t}}$, this also means that the above quantity depends only on these indices. Now we define the operators $T_{a_{s}, a_{t}}( \pm)$ by using these $R$ 's. Since now the entries of these new operators depend only on indices $a$ 's, we shall reduce $W_{i}$ to $V_{i}$ and the representation space $W_{j}$ to $V_{j}$. The action of $T_{a_{s}, a_{t}}( \pm)$ is defined as

$$
T_{a_{s}, a_{t}}( \pm)\left(\phi_{a_{u}}\right)=\sum_{a_{v}} B( \pm)_{a_{s} a_{u}, a_{v} a_{t}} \phi_{a_{c}}
$$

and the coproduct

$$
\Delta\left(T_{a_{s}, a_{t}}( \pm)\right)=\sum_{a_{s^{\prime}}} T_{a_{s}, a_{s^{\prime}}}( \pm) \otimes T_{a_{s^{\prime}}, a_{t}}( \pm) .
$$

Again we have equations similar to $(8.8)$ with $R$-matrix defined by

$$
R_{a_{s} a_{t}, a_{u} a_{v}}=B_{a_{s} a_{t}, a_{v} a_{u}},
$$


here all indices $a$ are in $V_{i}$. In this way, we found a set of Hopf algebras (when $i$ varies) with the same representation spaces.

It is not hard to show that the generators of the vertex quantum group commute with the intertwiners $K$ 's defined before. This is a consequence of the pentagon identity after taking the limit. We present the details in Appendix B. Note that the $K$ 's define the functor from the Moore-Seiberg category to the category of vector spaces (see Appendix B). In the spirit of [19] one actually reconstructs the vertex quantum group.

For example, let us consider the WZW model with group $S U(N)$. Let $i$ be the fundamental representation. Now the Hopf algebra defined before is just $U_{q}(S U(N))$. We thus extend the consideration for $S U(2) \mathrm{WZW}$ model in [12] to more general cases. An example for $S U(2) \mathrm{WZW}$ model is given below, where we find that the vertex quantum group is actually $U_{q}(S U(2))$. Finally, note that our presentation is different from the one in [12], where the generators $T_{\mu}$, the deformations of the generators of Lie algebra are defined. These operators generate the algebra, loosely speaking, dual to $U_{q}(S U(2))$.

Our discussion also reveals the relationship between the quantum group considered here and the IRF quantum group introduced in the last subsection. Obviously. further study is needed in order to understand the relation among various quantum groups better. And one also hopes to avoid using the lassoing procedure, since we cannot presumably assume that there is a set of solutions parametrized by some discrete parameters to the polynomial equations.

Examples. We shall show that the lassoing procedure actually works in several cases. First let us consider $S U(2) \mathrm{WZW}$ model. The main thing we want to calculate is $\lim _{k_{s} \rightarrow \infty} B_{s u, v t}$, which is called $R_{a_{s} a_{u}, a_{t} a_{v}}$, and plays the key role in the defining relation (8.8) of the quantum group. Let all $a$ 's be in the fundamental representation, there are just two possible values, $1 / 2$ and $-1 / 2$. The limit of $B_{s u, v t}$ in this case can be represented by the graph

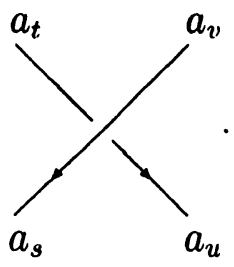

All braiding matrices are known in this case. What we need can be found, for example, in Witten [7]

$$
\begin{gathered}
B_{s l}\left[\begin{array}{ll}
1 & 1 \\
j & i
\end{array}\right]=q^{1 / 4} \delta_{s l} N_{1 j s} N_{1 s i} \frac{S_{0,1}}{S_{0,0}} \sqrt{\frac{S_{0, j}}{S_{0,0}}} \sqrt{\frac{S_{0, i}}{S_{0,0}}} \\
-q^{-1 / 4} \delta_{j i} N_{1 j s} N_{1 j l} \frac{S_{0,1}}{S_{0,0}} \sqrt{\frac{S_{0, s}}{S_{0,0}}} \sqrt{\frac{S_{0, l}}{S_{0,0}}} .
\end{gathered}
$$

Using this formula and substituting

$$
\frac{S_{0, i}}{S_{0,0}}=\sin \frac{2 i+1}{k+2} \pi / \sin \frac{\pi}{k+2},
$$


we find

$$
\begin{aligned}
R_{a_{s} a_{u}, a_{t} a_{v}}= & \lim _{k_{s} \rightarrow \infty} B_{s u, v t} \\
& =\left(q^{1 / 4} \delta_{a_{v}, a_{s}}-q^{-1 / 4} q^{\frac{a_{s}-a_{t}}{2}} \delta_{a_{v},-a_{t}}\right) \delta_{a_{v}, a_{s}+a_{u}-a_{t}},
\end{aligned}
$$

where we have used $|q|<1$, analytically extended from $q=\exp \left(\frac{i 2 \pi}{k+2}\right)$. The last delta-function in the above formula takes care of the conservation of weights. The $R$ matrix is a $4 \times 4$ matrix. It is illustrative to write it in the matrix form:

$$
R=q^{-1 / 4}\left(\begin{array}{cccc}
q^{1 / 2} & 0 & 0 & 0 \\
0 & -1 & 0 & 0 \\
0 & q^{1 / 2}-q^{-1 / 2} & -1 & 0 \\
0 & 0 & 0 & q^{1 / 2}
\end{array}\right)
$$

This matrix serves for the definition of $U_{q}(S U(2))$ as is well known. Some signs differ from the ones in the standard definition. These can be absorbed into a redefinition of the generators.

Next consider the rational torus. The index $i$ of the "integrable" representations of the current algebra runs in the set $Z_{2 k}$. The fusion rule is simply $i+j$ $=l \bmod 2 k$. By our lassoing prescription, there is only one independent vector in each space $V_{i}$. The weight is also denoted by $i . R_{a_{s} a_{u}, a_{t} a_{i}}$ can be non-vanishing only when $a_{s}=a_{t}=i$ and $a_{u}=a_{v}=j$. The relevant quantities in our calculation are

$$
B_{k_{s}, k_{s}+i-j}\left[\begin{array}{cc}
i & j \\
k_{s}+i & k_{s}-j
\end{array}\right]=e^{i \pi\left(h_{k_{s}+i}+h_{k_{s}-j}-h_{k_{s}+i-j}-h_{k_{s}}\right)} .
$$

Note that we have taken all non-vanishing elements of fusing matrices to be one. Using formula $h_{i}=i^{2} /(2 k)$, we find

$$
R_{i j, i j}=q^{i j}
$$

where $q=\exp \left(\frac{2 \pi i}{2 k}\right)$. The relevant quantity in defining the quantum group is $R_{11,11}=q$. But note that this matrix is just a number so the defining relation (8.8) for the generator does not give rise to any constraint. Therefore we learned that the quantum group is just the algebra generated by a single free generator $T$ together with its inverse. So any irreducible representation space is one dimensional. The deformation parameter $q$ plays no role in the structure of the quantum group. However, we suppose that for the fundamental representation $T|1\rangle=q|1\rangle$. Now representation $|j\rangle$ is constructed by taking the $j^{\text {th }}$ tensor product of $|1\rangle$. Since the co-product is given by $\Delta(T)=T \otimes T$, the action of $T$ on $|j\rangle$ is just $T|j\rangle=q^{j}|j\rangle$, compatible with (8.30) and formula (8.37) when we take $i=1$. Here we encounter a special case that the deformation parameter $q$ enters only in representations, each set of representations is characterized by a complex number $q$. Different sets are disconnected in the sense that any representation in one set does not appear in the tensor products of representations of another set. Our quantum group presented here is different from the one in [10].

Our calculation for $S U(2)$ WZW model can be easily extended to the general case of $S U(N)$, where relevant $B$ 's have been calculated, see for example Witten [7]. 


\section{A. Quasitriangular Hopf Algebras}

In this appendix we describe the exact definition of a quasitriangular Hopf algebra $[8,18]$ and work out the universal $\mathscr{R}$-matrix for the IRF quantum group. Similar consideration applies to the vertex quantum group.

A Hopf algebra $A$ is an associative algebra with a unit $\eta$ and the product ., together with the coproduct $\Delta$, the antipode $S$ and the counit $\varepsilon$. The product · is an algebra map from $A \otimes A$ to $A$ and the unit may be viewed as a map $\eta$ from the complex numbers $C$ to $A$. Conversely, the coproduct is an algebra map from $A$ to $A \otimes A$ and the counit is a map from $A$ to $C$. The antipode, as the reminiscence of the inverse in group theory, is a map from $A$ to itself. Now we require that the coproduct is associative, namely we must have $(\mathrm{id} \otimes \Delta) \otimes \Delta=(\Delta \otimes \mathrm{id}) \otimes \Delta$, where id is the identity map from $A$ to itself. For the counit, we have $\varepsilon(1)=1$. The antipode is an anti-algebra map, namely we have $S(a b)=S(b) S(a)$. Besides, we have the consistency condition

$$
\cdot(S \otimes \mathrm{id}) \Delta=\cdot(\operatorname{id} \otimes S) \Delta=\eta \varepsilon .
$$

This completes our definition for a Hopf algebra [8]. In Subsect. 8.1 we have defined the coproduct and the counit for the generators of the IRF quantum group, the definitions are extended to the whole algebra by the morphism property of these maps. It is easy to see that the definitions for the antipode and the counit in (8.16) follow directly from the above consistency condition.

We define the quasitriangular Hopf algebra. Consider the Hopf algebra $A$. Let $\mathscr{R}$ be an element in $A \otimes A$, having an inverse, and let $\tau$ be the twist map from $A \otimes A$ to itself. If the following identities

$$
\begin{gathered}
(\Delta \otimes \mathrm{id}) \mathscr{R}=\mathscr{R}_{13} \mathscr{R}_{23}, \quad(\mathrm{id} \otimes \Delta) \mathscr{R}=\mathscr{R}_{13} \mathscr{R}_{12}, \\
\tau \Delta(a)=\mathscr{R}(\Delta(a)) \mathscr{R}^{-1}, \quad \forall a \in A,
\end{gathered}
$$

are satisfied, then $A$ is a quasitriangular Hopf algebra. Above we use the notation $\mathscr{R}_{i j}$ to denote the corresponding $\mathscr{R}$ taking values in the $i^{\text {th }}$ copy tensoring $j^{\text {th }}$ copy of $A$.

To find the universal $\mathscr{R}$-matrix for the Hopf algebra $A$ defined in Subsect. 8.1, we follow the method in [18]. Associated to $A$, we can define the dual Hopf algebra $A^{*}$ generated by generators $U_{s t}$. We define the following pairing:

$$
\begin{aligned}
& \left\langle U_{s t}, T_{s^{\prime} t^{\prime}}(+)\right\rangle=R(+)_{s s^{\prime}, t t^{\prime}}=B(+)_{s s^{\prime}, t^{\prime} t}, \\
& \left\langle U_{s t}, T_{s^{\prime} t^{\prime}}(-)\right\rangle=R(-)_{s s^{\prime}, t t^{\prime}}=B(-)_{s s^{\prime}, t^{\prime} t} .
\end{aligned}
$$

Again these generators are subject to the identity

$$
R(+)(U \otimes 1)(1 \otimes U)=(1 \otimes U)(U \otimes 1) R(+),
$$

where we used the same notation in (8.8).

The algebra generated by $U_{s t}$ is also a Hopf algebra. To see this, let us define the product, coproduct, counit, and antipode by the following requirements:

$$
\begin{gathered}
\langle u v, a\rangle=\langle u \otimes v, \Delta(a)\rangle, \quad\langle\Delta(u), a \otimes b\rangle=\langle u, a b\rangle, \\
\varepsilon(u)=\langle u, 1\rangle, \quad\langle S(u), a\rangle=\langle u, S(a)\rangle,
\end{gathered}
$$

where $u, v \in A^{*}$ and $a, b \in A$. 
If there exists a universal $\mathscr{R}$-matrix for $A$, it can be viewed as a map from $A^{*}$ to $A$ by the pairing. By the general construction [18], we can prove that the universal $\mathscr{R}$-matrix is given by the map $\mathscr{R}: U_{s t} \rightarrow T_{s t}(+)$, or

$$
\mathscr{R}=\sum_{s t, u v} R_{1}^{s t, u v} T_{s t}(+) \otimes T_{u v}(-),
$$

where $R_{1}$ satisfies

$$
\sum_{x y} R_{1}^{s t, x y} R(+)_{u x, v y}=\sum_{x y} R_{1}^{s t, x y} B(+)_{u x, y v}=\delta_{s u} \delta_{t v} .
$$

It is not hard to see that $R_{1}$ exists and is uniquely determined by the above equation, when we use the tetrahedron symmetry to write $B(+)_{u x, y v}$ in terms of $B(+)_{x y, u v}$. The explicit solution can be written in terms of $B$ but we will not write it down here.

As for the universal $\mathscr{R}$-matrix for the vertex quantum group, we can either find it by applying a method similar to the one used here for the algebra generated by $T_{a b}$, or by applying the lassoing method to the universal $\mathscr{R}$-matrix obtained above.

\section{B. Moore-Seiberg Category}

We briefly review the definition of the Moore-Seiberg category for a rational conformal field theory in this appendix. Indeed mathematicians have studied the similar category for some time [20], they call this kind of category the rigid abelian quasitensor category. However, we prefer to call the category associated with a RCFT the Moore-Seiberg category for the following reason: usually the rigid abelian quasitensor category defined by mathematicians has infinitely many building blocks. Namely the number of elementary objects is infinite. This is because there is a functor from this category to the category of vector spaces. For a RCFT, we have finitely many elementary objects and we need modify the notation of the category of vector spaces, since usually the notion of the restricted tensor product is needed.

For the definition of the category and abelian quasitensor category, see [3,18]. The Moore-Seiberg category is built from the elementary objects $S_{i}$. Here to each highest weight $i$ of the chiral algebra, we associated an object $C_{i}$. We need not assume there is a chiral algebra here, we just suppose that we have a set (i). Abstractly, we define the morphism set $\operatorname{Mor}\left(S_{i}, S_{j}\right)$ contains only one morphism $O_{j i}$, and the abelian group is defined by $O_{j i}+O_{j i}=O_{j i}$. Define various tensor products of the basic objects, and the morphisms are given by tensoring $O$ 's. One also defines the tensor product of the objects with vector spaces. This enlarges the set of objects. Now we impose a condition on the tensor product by

$$
S_{i} \otimes S_{j}=\otimes_{k} W_{i j}^{k} \otimes S_{k} .
$$

Here the vector space is the one defined in Sect. 5. As a quasitensor category, we shall define the map $F$

$$
S_{i} \otimes\left(S_{j} \otimes S_{k}\right) \stackrel{\mathbb{E}}{\cong}\left(S_{i} \otimes S_{j}\right) \otimes S_{k},
$$

this is provided by the map

$$
\oplus_{m} W_{i m}^{l} \otimes W_{j k}^{m} \stackrel{F}{\cong} \oplus_{n} W_{n k}^{l} \otimes W_{i j}^{n} .
$$


In addition these $F$ 's must satisfy the pentagon identity. We should have another map $\Omega: S_{i} \otimes S_{j} \rightarrow S_{j} \otimes S_{i}$, and this is provided by $\Omega: W_{i j}^{k} \rightarrow W_{j i}^{k} . \Omega$ must satisfy two hexagon identities. Our category is quasitensor because usually we have not $\Omega^{2}=1$, instead this is a pure phase. To cast the category into a rigid one, we must have an object $\operatorname{Hom}(X, Y)$ for a pair objects $(X, Y)$. This is achieved by letting $S_{i}^{\vee}=\operatorname{Hom}\left(S_{i}, 1\right)$ and $\operatorname{Hom}\left(S_{i}, S_{j}\right)=S_{j} \otimes S_{i}{ }^{\vee}$. Now we have the Moore-Seiberg category. So far it is an abstract notion, although in defining it we need some practical objects such as the maps $F, \Omega$. These maps contain the whole knowledge of the duality data. Moore and Seiberg have been able to define the rank of $S_{i}$ abstractly; the striking point is that this rank is given by $1 / F_{i}$. It should be interesting to explain the formula $\mathrm{M} 2$ for $S(j)$ in this way.

Now if we have a vector space $V_{i}$ to each $S_{i}$, and for these spaces we can define the restricted tensor product

$$
V_{i} \otimes V_{j} \cong \oplus_{k} W_{i j}^{k} \otimes V_{k},
$$

then the map $\omega: S_{i} \rightarrow V_{i}$ constitutes a functor from the Moore-Seiberg category to the category of vector space equipped with the restricted product. Since for these vector spaces we also have maps $F, \Omega$ which are well-defined in terms of the restricted tensor product, $\omega$ is called as the forgetful functor.

Majid in a recent paper [19] showed that one can reconstruct the quantum group by the forgetful functor $\omega$. However the tensor product of the vector spaces are not the restricted one, namely the dimensions of the vector spaces must be conserved. It is not hard to generalize Majid's result to the restricted case. The main point in the reconstruction is that a generator $T$ of the quantum group is defined by a set of linear maps $T_{i}: V_{i} \rightarrow V_{i}$, which are covariant in the sense of [19], with an appropriate notion of coproduct $\Delta$ such that the following diagram is commutative:

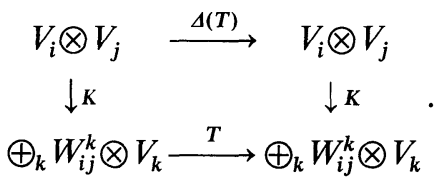

Thus, the key point is that we must have a set of $K$ 's, namely the Clebsch-Gordan coefficients. This can be easily extended to the restricted case.

We found that actually we still do not have the reconstruction theorem for the quantum group, since usually we do not know how to extract CG coefficients from the duality data. However, in Sect. 8, we show that for the WZW models, or more generally for those models for which one can perform Witten's lassoing, one can construct these desired CG coefficients. Also we constructed the generators explicitly. We show here that these generators and CG coefficient satisfy the above commutative diagram. For simplicity, let us assume that $N_{i j}^{k} \leqq 1$. Consider $\Delta\left(T_{a_{s}, a_{t}}( \pm)\right)$ acting on $\phi_{a_{u}} \otimes \phi_{a_{v}}$, where $\phi_{a_{u}} \in V_{i}$ and $\phi_{a_{v}} \in V_{j}$. (B.5) implies

$$
\sum_{a_{s^{\prime}}, a_{u^{\prime}}, a_{v^{\prime}}} B_{a_{s} a_{u}, a_{u^{\prime}} a_{s^{\prime}}} B_{a_{s} a_{v^{\prime}}, a_{v^{\prime}} a_{t}} K_{k a_{w}}^{i a_{u^{\prime}}, j a_{v^{\prime}}}=\sum_{a_{w^{\prime}}} \boldsymbol{B}_{a_{s} a_{w^{\prime}}, a_{w^{\prime}} a_{t}} K_{k a_{w^{\prime}}}^{i a_{u}, j a_{v}},
$$

where $a_{w}$ and $a_{w^{\prime}}$ are indices in $V_{k}$.

To prove (B.6), note that the commutative diagram for the IRF quantum group in 8.12 implies

$$
\sum_{s, u^{\prime}, v^{\prime}} B_{s u, u^{\prime} s^{\prime}} B_{s^{\prime} v, v^{\prime} t} F_{u^{\prime} v^{\prime}}^{w}=\sum_{w^{\prime}} B_{s w^{\prime}, w t} F_{u v}^{w^{\prime}}
$$


where

$$
F_{u v}^{w}=F_{j v k}\left[\begin{array}{cc}
i & j \\
j_{u} & k
\end{array}\right]\left(F_{i} F_{j} F_{k}\right)^{-1 / 4} .
$$

It is not hard to see that (B.7) is equivalent to the pentagon identity. Now after taking the infinite limit in (B.7), all the B's go to B's in (B.6). By the tetrahedron symmetry and the definition for the CG coefficients, we find that

$$
\lim _{j_{w} \rightarrow \infty} F_{u^{\prime} v^{\prime}}^{w}=\left(F_{k}\right)^{-1 / 2} K_{k a_{w}}^{i a_{u^{\prime}}, j a_{v^{\prime}}} \text {. }
$$

So multiplying a factor $\left(F_{k}\right)^{1 / 2}$ to both sides of (B.7) and taking the infinite limit, we reach (B.6).

In Sect. 8, we mentioned that the CG coefficients and the $F$-matrix satisfy the desired equations. Thus, by construction, we have a functor $\omega$ from the MooreSeiberg category to the category of vector spaces. Here we showed that the generators defined in Sect. 8 commute with the intertwiners $K$, by the reconstruction theorem in [19]. These generators are the ones for the quantum group. To summarize, we give the following theorem.

Theorem. If all those limits defined in Sect. 8 exist, then actually we have reconstructed generators of the quantum group by (8.30) and $\mathrm{CG}$ coefficients in the representation theory of the quantum group by (8.21).

Acknowledgements. I would like to thank V. Fateev, J.L. Petersen, Y.-S. Wu, and M. Yu for useful discussions. I am grateful to J. L. Petersen for his careful reading of the manuscript. Finally, I would like to thank J. Fröhlich and another referee for valuable suggestions on revising this paper.

\section{References}

1. Belavin, A.A., Polyakov, A.M., Zamolodchikov, A.B.: Infinite conformal symmetry in two dimensional quantum field theory. Nucl. Phys. B 241, 33 (1984)

2. Verlinde, E.: Fusion rules and modular transformations in 2-D conformal field theory. Nucl. Phys. B 300, 360 (1988)

Dijkgraaf, R., Verlinde, E.: Modular invariance and the fusion algebra. In the Proceedings of the Annecy Conference on Conformal Field Theory. Nucl. Phys. B (Proc. Suppl.) 5 B (1988)

3. Moore, G., Seiberg, N.: Polynomial equations for rational conformal field theories. Phys. Lett. 212 B, 451 (1988); Classical and quantum conformal field theory. Commun. Math. Phys. 123, 177 (1989)

4. Fröhlich, J.: Statistics of fields, the Yang-Baxter equation, and the theory of knots and links. Lectures and Cargese 1987

Rehren, K.-H., Schroer, B.: Einstein causality and Artin braids. Nucl. Phys. B 312, 715 (1989) Schroer, B.: Quasiprimary fields: an approach to positivity of 2-D conformal quantum field theory. Nucl. Phys. B 295, 4 (1988)

Rehren, K.-H.: Locality of conformal fields in two dimensions: Exchange algebra on the light cone. Commun. Math. Phys. 116, 675 (1988)

5. $\mathrm{Li}, \mathrm{M}$., Yu, M.: Braiding matrices, modular transformations and topological field theories in $2+1$ dimensions, Commun. Math. Phys. 127, 195 (1990); Modular transformations of conformal blocks in WZW models on Riemann surfaces of higher genus. Phys. Lett. 241 B, $522(1990)$

6. Li, M., Yu, M.: Duality ensures modular covariance. Phys. Lett. B 249, 422 (1990)

7. Witten, E.: Quantum field theory and the Jones polynomials. Commun. Math. Phys. 121, 351 (1989); Gauge theories and integrable lattice models. Nucl. Phys. B 322, 629 (1989) 
8. Drinfel'd, V.G.: Quantum groups. In: Proceedings of the International Congress of Mathematicians, Berkeley Cal. (1986)

Jimbo, M.: Lett. Math. Phys. 10, 63 (1985)

Faddeev, L., Reshetikhin, N.Yu., Takhtatajan, L.A.: LOMI preprint, E-14-87 (1987)

Kirillov, A.N., Reshetikhin, N.Yu.: Representations of the algebra $U_{q}(s l(2))$. $q$-orthogonal polynomials and invariants of links. Preprint LOMI-E-9-88 (1988)

9. Kohno, T.: Monodromy representation of the braid group and Yang-Baxter equations. In: International conference in homor of J. K. Kozul (1987)

Tsuchiya, A., Kanie, Y.: Vertex operators in the conformal field theory on $P^{1}$ and monodromy representations of the braid group. Lett. Math. Phys. 13, 303 (1987); In: Conformal field theory and solvable lattice models. Adv. Stud. Pure Math. 16, 297 (1988)

10. Alvarez-Gaumé, L., Gomez, C., Sierra, G.: Quantum group interpretation of some conformal field theories. Phys. Lett. 220 B, 142 (1989); duality and quantum groups. Nucl. Phys. B 330, 347 (1990)

11. Moore, G., Reshetikhin, N.: A comment on quantum group symmetry in conformal field theory. Nucl. Phys. B 328, 557 (1989)

12. Witten, E.: Gauge theories, vertex models, and quantum groups. Nucl. Phys. B 330, 285 (1990)

13. Alvarez-Gaumé, L., Gomez, C., Sierra, G.: Hidden quantum symmetries in rational conformal field theories. Nucl. Phys. B 319, 155 (1989)

14. Fröhlich, J., King, C.: Two-dimensional conformal field theory and three dimensional topology. Int. J. Mod. Phys. A 4, 5321 (1989)

15. Felder, G., Fröhlich, J., Keller, G.: On the structure of unitary conformal field theory. I. Existence of conformal blocks. Commun. Math. Phys. 124, 417 (1989); on the structure of unitary conformal field theory. II. Representation theoretic approach. Commun. Math. Phys. 130, 1 (1990)

16. Gao, Y.H., Li, M., Yu, M.: Fusion algebra and fusing matrices. ICTP preprint, IC/89/316. Submitted to Int. J. Mod. Phys. A

17. Moore, G., Seiberg, N.: Naturality in conformal field theory. Nucl. Phys. B 313, 16 (1989) Brustein, R., Yankielowicz, S., Zuber, J.-B.: Factorization and selection rules of operator product algebras in conformal field theory. Nucl. Phys. B 313, 321 (1989)

18. Majid, S.: Quasitriangular Hopf algebras and Yang-Baxter equations. Int. J. Mod. Phys. A 5, 1 (1990)

19. Majid, S.: Reconstruction of rational conformal field theories. DAMTP/89-40 (1989)

20. Deligne, P., Milne, J.S.: Tannakian categories. Lecture Notes in Math., vol. 900. Berlin, Heidelberg, New York: Springer 1982; Lyubashenko, V.V.: Usp. Math. Nauk. 41, 185 (1986)

21. Li, M.: Work in progress

22. Dijkgraaf, R., Verlinde, E., Verlinde, H.: Commun. Math. Phys. 115, 649 (1988)

23. Moore, G., Seiberg, N.: Taming the conformal zoo. Phys. Lett. 220 B, 422 (1989)

24. Dijkgraaf, R.. Witten, E.: Topological gauge theories and group cohomology. Preprint IASSNS-HE-89/33 (1989)

25. Cappelli, A., Itzkyson, C., Zuber, J.-B.: The A-D-E classification of minimal and $A_{1}^{(1)}$ conformal invariant theories. Commun. Math. Phys. 113, 26 (1987)

Bauer, M., Itzykson, C.: Modular transformations of $S U(N)$ affine characters and their commutant. Commun. Math. Phys. 127, 617 (1990) 
